\title{
Linearized radiative transfer models for retrieval of cloud parameters from EPIC/DSCOVR measurements
}

\author{
Víctor Molina García*, Sruthy Sasi, Dmitry S. Efremenko, Adrian Doicu, Diego Loyola \\ Deutsches Zentrum für Luft- und Raumfahrt (DLR), Institut für Methodik der Fernerkundung (IMF), 82234 Oberpfaffenhofen, Germany
}

\begin{abstract}
In this paper, we describe several linearized radiative transfer models which can be used for the retrieval of cloud parameters from EPIC (Earth Polychromatic Imaging Camera) measurements. The approaches under examination are (1) the linearized forward approach, represented in this paper by the linearized discrete ordinate and matrix operator methods with matrix exponential, and (2) the forward-adjoint approach based on the discrete ordinate method with matrix exponential. To enhance the performance of the radiative transfer computations, the correlated $k$-distribution method and the Principal Component Analysis (PCA) technique are used. We provide a compact description of the proposed methods, as well as a numerical analysis of their accuracy and efficiency when simulating EPIC measurements in the oxygen A-band channel at $764 \mathrm{~nm}$. We found that the computation time of the forwardadjoint approach using the correlated $k$-distribution method in conjunction with PCA is approximately $13 \mathrm{~s}$ for simultaneously computing the derivatives with respect to cloud optical thickness and cloud top height.
\end{abstract}

Keywords: inversion, linearized models, adjoint radiative transfer, EPIC

\section{Introduction}

EPIC (Earth Polychromatic Imaging Camera) is a 10-channel spectroradiometer $(317-780 \mathrm{~nm})$ onboard the spacecraft DSCOVR (Deep Space Climate Observatory), and was designed to measure the atmosphere and surface properties over the whole sunlit half of the Earth from the Lagrange point $\mathrm{L}_{1}$. DSCOVR provides observations of the Earth at near backward directions with scattering angles ranging from $168^{\circ}$ to $176^{\circ}$. EPIC has two pairs of reference and absorption channels in the oxygen A-band (780 and $764 \mathrm{~nm}$ ) and B-band (680 and $688 \mathrm{~nm}$ ), which are used for monitoring the vegetation condition [1], the aerosol layer height and optical depth [2], as well as the cloud height and optical depth [3]. A further description of EPIC/DSCOVR geometry and their channels can be found in [4].

In [4], we analyzed exact and approximate radiative transfer models regarding their applicability to the retrieval of cloud parameters from EPIC measurements. It has been shown that the exact Discrete Ordinate method with Matrix Exponential (DOME) and the Matrix Operator method with Matrix Exponential (MOME) using the correlated $k$-distribution method [5] in conjunction with the Principal Component Analysis (PCA) technique [6-9] fulfill the accuracy and efficiency requirements for such kind of application.

However, the retrieval of atmospheric constituents from satellite measurements also requires the knowledge of weighting functions, i.e. the partial derivatives of the measured radiance with respect to the atmospheric parameters being retrieved. The process of obtaining this set of partial derivatives,

\footnotetext{
${ }^{*}$ Corresponding author

Email address: Victor.MolinaGarcia@dlr.de (Víctor Molina García)
}

which constitute the matrix of weighting functions or Jacobian, is commonly referred to as linearization analysis. There are two common linearization approaches: the linearized forward approach and the forward-adjoint approach.

1. In the linearized forward approach, the partial derivatives are computed analytically. Such linearized radiative transfer models based on the conventional discrete ordinate method and the matrix operator method have been developed by Spurr [10-13].

2. In the forward-adjoint approach, the measured radiance is expressed as the scalar product of the solution of the adjoint problem and the source function of the forward problem. Using the linearization technique to the forward and adjoint problems (i.e. using the differentiation operator by means of the chain rule), analytical expressions for the weighting functions have been derived in [14-20] and also compared to other methods in $[21,22]$. The forwardadjoint approach is extremely efficient because only two radiative transfer calculations are required for computing the derivatives.

In this paper, we discuss the linearization of these radiative transfer models for EPIC retrieval purposes. More precisely, we will apply the linearized forward approach to DOME and MOME, and design a forward-adjoint approach based on DOME. As for the radiance calculations, the performances of the derivative calculations will be enhanced using acceleration techniques. The accuracy and efficiency of the linearized radiative transfer models will be analyzed by performing simulations which are pertinent to the retrieval of cloud parameters from EPIC oxygen A- and B-band measurements. 
The paper is organized as follows. In Section 2 we review the radiative transfer models DOME and MOME, in Section 3 we discuss the linearized radiative transfer models, and in Section 4 we present runtime and error analyses for these models. The results are summarized in Section 5. Some specific features of the linearization procedure are outlined in the appendices.

\section{Radiative transfer models}

The radiative transfer equation for the total radiance $I(r, \mathbf{\Omega})$ at point $r$ and in the direction $\mathbf{\Omega}=(\mu, \varphi)$ reads as

$$
\begin{aligned}
\mu \frac{\mathrm{d} I}{\mathrm{~d} r}(r, \boldsymbol{\Omega}) & =-\sigma_{\mathrm{ext}}(r) I(r, \boldsymbol{\Omega}) \\
& +\frac{\sigma_{\mathrm{sct}}(r)}{4 \pi} \int_{4 \pi} P\left(r, \boldsymbol{\Omega}, \boldsymbol{\Omega}^{\prime}\right) I\left(r, \boldsymbol{\Omega}^{\prime}\right) \mathrm{d} \Omega^{\prime},
\end{aligned}
$$

where $\sigma_{\text {ext }}$ and $\sigma_{\text {sct }}$ are the extinction and scattering coefficients, respectively, and $P$ is the scattering phase function. The phase function $P$ is expanded in terms of normalized Legendre polynomials $P_{n}$, i.e.

$$
P\left(r, \boldsymbol{\Omega}, \mathbf{\Omega}^{\prime}\right)=P(r, \cos \Theta)=\sum_{n=0}^{\infty} c_{n} \chi_{n}(r) P_{n}(\cos \Theta),
$$

where $c_{n}=\sqrt{(2 n+1) / 2}$ and $\cos \Theta=\boldsymbol{\Omega} \cdot \boldsymbol{\Omega}^{\prime}$. The differential equation (1) is endowed with the top-of-atmosphere (TOA) boundary condition $\left(r=r_{\mathrm{TOA}}\right)$,

$$
I\left(r_{\mathrm{TOA}}, \mathbf{\Omega}^{-}\right)=F_{0} \delta\left(\mathbf{\Omega}^{-}-\mathbf{\Omega}_{0}\right),
$$

and the surface boundary condition $\left(r=r_{\mathrm{s}}\right)$,

$$
I\left(r_{\mathrm{s}}, \mathbf{\Omega}^{+}\right)=\frac{A}{\pi} \int_{2 \pi} \rho\left(\mathbf{\Omega}^{+}, \mathbf{\Omega}^{-}\right)\left|\mu^{-}\right| I\left(r_{\mathrm{s}}, \mathbf{\Omega}^{-}\right) \mathrm{d} \Omega^{-},
$$

where $F_{0}$ is the incident solar flux, $\boldsymbol{\Omega}_{0}=\left(-\mu_{0}, \varphi_{0}\right)$ with $\mu_{0}>0$ is the incident solar direction, and $A$ and $\rho$ are the surface albedo and the normalized bi-directional reflection function, respectively. The notations $\boldsymbol{\Omega}^{+}$and $\boldsymbol{\Omega}^{-}$stand for an upward and a downward direction, respectively. The total radiance is decomposed into the diffuse radiance $I_{\mathrm{d}}(r, \boldsymbol{\Omega})$ and the direct solar beam $I_{\odot}(r, \boldsymbol{\Omega})$, i.e.

$$
I(r, \boldsymbol{\Omega})=I_{\mathrm{d}}(r, \boldsymbol{\Omega})+I_{\odot}(r, \boldsymbol{\Omega})
$$

where

$$
I_{\odot}(r, \boldsymbol{\Omega})=F_{0} \delta\left(\boldsymbol{\Omega}-\mathbf{\Omega}_{0}\right) T(r),
$$

$T(r)=\exp \left[-\tau_{\text {ext }}\left(\left|\mathbf{r}-\mathbf{r}_{\mathrm{TOA}}\right|, \mathbf{\Omega}_{0}\right)\right]$ is the solar transmission, and $\tau_{\text {ext }}\left(\left|\mathbf{r}-\mathbf{r}_{\mathrm{TOA}}\right|, \boldsymbol{\Omega}_{0}\right)$ is the solar optical depth between a general point $\mathbf{r}$ and the specific point at the top of the atmosphere $\mathbf{r}_{\mathrm{TOA}}$ in a spherical atmosphere.

Assuming cosine-azimuthal expansions for the diffuse radiance, the scattering phase function and the normalized bi-directional reflection function, we formulate a boundary-value problem for each Fourier component of the diffuse radiance $I_{\mathrm{d} m}(r, \mu)$ by means of the discrete ordinate method. The angular variation of the phase function and radiance is discretized in $M$ discrete-ordinate directions per hemisphere, and the set of GaussLegendre quadrature points and weights in the interval $(0,1)$ is denoted by $\left\{\mu_{k}, w_{k}\right\}_{k=1}^{M}$. The atmosphere is discretized in $N$ levels: $r_{1}=r_{\mathrm{TOA}}>r_{2}>\ldots>r_{N}=r_{\mathrm{s}}$, and a layer $j$, bounded above by the level $r_{j}$ and below by the level $r_{j+1}$, has the geometrical thickness $\Delta r_{j}=r_{j}-r_{j+1}$. The extinction and scattering coefficients, as well as the phase function coefficients, are assumed to be constant within each layer; their average values on layer $j$ are $\sigma_{\text {ext } j}, \sigma_{\text {sct } j}$ and $\chi_{n j}$, respectively.

The radiative transfer models analyzed in [4] are DOME and MOME. In summary, these models can be characterized as follows:

1. DOME $[23,24]$ relies on the layer equation, which relates the level values of the radiance field $\mathbf{i}_{m j}=\left[\mathbf{i}_{m j}^{+} ; \mathbf{i}_{m j}^{-}\right]^{\top}$ with $\left[\mathbf{i}_{m j}^{ \pm}\right]_{k}=I_{\mathrm{d} m}\left(r_{j}, \pm \mu_{k}\right), k=1, \ldots, M$, that is,

$$
\mathrm{A}_{m j}^{1} \mathbf{i}_{m j}+\mathrm{A}_{m j}^{2} \mathbf{i}_{m j+1}=\mathrm{b}_{m j}
$$

The layer equation together with the boundary conditions at the top and the bottom of the atmosphere, as well as the continuity of the radiance across layers, are assembled into the global matrix of the entire atmosphere, and the solution of the resulting system of equations yields the level values of the diffuse radiance field.

2. MOME applies to a molecular atmosphere containing a homogeneous cloud placed between the top level $r_{j_{\min }}$ and the bottom level $r_{j_{\max }+1}$. The method is a combination of the conventional matrix operator method and the discrete ordinate method with matrix exponential, in the sense that the reflection matrix and vector of the atmosphere below the cloud top are computed in the framework of the conventional matrix operator method, while the level values of the diffuse radiance field above the cloud top are computed in the framework of the discrete ordinate method with matrix exponential. The conventional matrix operator method, e.g. [25], uses the interaction principle equation

$$
\left[\begin{array}{c}
\mathbf{i}_{m j}^{+} \\
\mathbf{i}_{m j+1}^{-}
\end{array}\right]=\left[\begin{array}{cc}
\mathbf{R}_{m j} & \mathbf{T}_{m j} \\
\mathbf{T}_{m j} & \mathbf{R}_{m j}
\end{array}\right]\left[\begin{array}{c}
\mathbf{i}_{m j}^{-} \\
\mathbf{i}_{m j+1}^{+}
\end{array}\right]+\left[\begin{array}{c}
\boldsymbol{\Sigma}_{m j}^{+} \\
\boldsymbol{\Sigma}_{m j}^{-}
\end{array}\right],
$$

where $\mathbf{R}_{m j}$ and $\mathbf{T}_{m j}$ are the reflection and transmission matrices of layer $j$, respectively, and $\boldsymbol{\Sigma}_{m j}^{ \pm}$is the source vector. The computation process is an upward recurrence over the atmospheric layers [13]: If $\mathbf{R}_{m j+1}$ and $\mathbf{r}_{m j+1}$ are respectively the reflection matrix and the reflection vector of stack $j+1$ (the group of layers bounded above by the level $r_{j+1}$ and below by the surface level $r_{N}$ ), the interaction principle equation for stack $j$ (the group of layers bounded above by the level $r_{j}$ and below by the surface level $r_{N}$ ) reads as

$$
\mathbf{i}_{m j}^{+}=\mathrm{R}_{m j} \mathbf{i}_{m j}^{-}+\mathrm{r}_{m j},
$$

where $\mathrm{R}_{m j}$ and $\mathrm{r}_{m j}$ are computed with the adding formulas

$$
\begin{aligned}
\mathbf{R}_{m j} & =\mathbf{R}_{m j}+\mathbf{T}_{m j} \mathbf{R}_{m j+1}\left(\mathbf{I}-\mathbf{R}_{m j} \mathbf{R}_{m j+1}\right)^{-1} \mathbf{T}_{m j} \\
\mathbf{r}_{m j} & =\Sigma_{m j}^{+}+\mathbf{T}_{m j} \mathbf{r}_{m j+1} \\
& +\mathbf{T}_{m j} \mathbf{R}_{m j+1}\left(\mathbf{I}-\mathbf{R}_{m j} \mathbf{R}_{m j+1}\right)^{-1}\left(\mathbf{R}_{m j} \mathbf{r}_{m j+1}+\Sigma_{m j}^{-}\right)
\end{aligned}
$$


The two methods are combined by considering the interaction principle equation at the cloud top, i.e.

$$
\mathbf{i}_{m j_{\min }}^{+}=\mathrm{R}_{m j_{\min }} \mathbf{i}_{m j_{\min }}^{-}+\mathrm{r}_{m j_{\min }}
$$

as the surface boundary condition for the discrete ordinate method with matrix exponential.

The layer quantities $\mathrm{A}_{m j}^{1}, \mathrm{~A}_{m j}^{2}$ and $\mathbf{b}_{m j}$ in (7), as well as $\mathbf{R}_{m j}, \mathbf{T}_{m j}$ and $\boldsymbol{\Sigma}_{m j}^{ \pm}$in (8), are expressed in terms of the exponential of the layer matrix

$$
\mathbf{A}_{m j}=\left[\begin{array}{cc}
\mathbf{A}_{m j}^{11} & \mathbf{A}_{m j}^{12} \\
-\mathbf{A}_{m j}^{12} & -\mathbf{A}_{m j}^{11}
\end{array}\right],
$$

with entries

$$
\begin{aligned}
{\left[\mathbf{A}_{m j}^{11}\right]_{k l} } & =\frac{1}{2 \mu_{k}}\left[w_{l} \sigma_{\mathrm{sct} j} p_{m j}\left(\mu_{k}, \mu_{l}\right)-2 \sigma_{\mathrm{ext} j} \delta_{k l}\right] \\
{\left[\mathbf{A}_{m j}^{12}\right]_{k l} } & =\frac{1}{2 \mu_{k}} w_{l} \sigma_{\mathrm{sct} j} p_{m j}\left(\mu_{k},-\mu_{l}\right) .
\end{aligned}
$$

Note that the product $\sigma_{\mathrm{sct} j} p_{m j}\left(\mu, \mu^{\prime}\right)$ in (14) and (15) is computed as

$$
\begin{aligned}
\sigma_{\mathrm{sct} j} p_{m j}\left(\mu, \mu^{\prime}\right) & =\sum_{n=m}^{2 M-1} \xi_{n j} P_{n}^{m}(\mu) P_{n}^{m}\left(\mu^{\prime}\right), \\
\xi_{n j} & =\sigma_{\mathrm{sct} j} \chi_{n j},
\end{aligned}
$$

so that the optical input parameters of the radiative transfer models are $\sigma_{\text {ext } j}$ and $\xi_{n j}$ for $j=1, \ldots, N-1$ and $n=0, \ldots, 2 M-1$. DOME and MOME rely on the same matrix exponential formalism. The matrix exponential can be computed by employing the eigendecomposition method or the Padé approximation. In the first case, the layer quantities depend on the inverse of the eigenvector matrix $\mathbf{V}_{m j}$ and the eigenvalue matrix $\boldsymbol{\Lambda}_{m j}=\operatorname{diag}\left[\lambda_{1}, \ldots, \lambda_{M}\right]$ of $\mathbf{A}_{m j}$, while, in the second case, the layer quantities are expressed in terms of the layer matrix $\mathbf{A}_{m j}$ [4]. In the layer $j$, the matrix exponential is computed by means of the Padé approximation if $\left\|\mathbf{A}_{m j} \Delta r_{j}\right\| \leq 1$, and by the eigendecomposition method if this is not the case. For radiance calculations in the EPIC oxygen A-band absorption channel, DOME and MOME have been used in [4] together with the following acceleration techniques:

1. the delta-M [26] and the truncated-plus-single-scattering (TMS) method [27];

2. the telescoping technique $[12,23]$, which consists of the solution of a (reduced) boundary-value problem for the cloud layers and azimuthal modes $m>2$;

3 . the method of false discrete ordinate [25, 28-30], which eliminates the source integration in the post-processing step of the discrete ordinate method;

4. the correlated $k$-distribution method [5] and the PCA technique [6-9] (independently and together).

\section{Linearized radiative transfer models}

The radiance measured by the instrument is a function of various atmospheric and surface parameters of interest. In the first category we include, for example, the layer values of the trace gas extinction coefficient and the cloud parameters, while in the second category we include the surface albedo and parameters characterizing the normalized bi-directional reflection function. In the following, we consider the computation of the partial derivatives of the measured radiance with respect to a set of atmospheric parameters $\varsigma_{i}, i=1, \ldots, N_{\mathrm{p}}$, since the basic concepts are fully represented in this case. Before proceeding, we mention that the optical input parameters of the linearized radiative transfer models are $\partial \sigma_{\text {ext } j} / \partial \varsigma_{i}$ and $\partial \xi_{n j} / \partial \varsigma_{i}$ for $i=1, \ldots, N_{\mathrm{p}}$, $j=1, \ldots, N-1$, and $n=0, \ldots, 2 M-1$.

\subsection{Linearized forward approach}

A detailed description of the linearized forward approach can be found in [10-12]. In our case, the linearized forward approach is applied to DOME and MOME. To compute the partial derivative with respect to the atmospheric property $\varsigma_{i}$, we proceed as follows:

1. In DOME, we linearize the layer equation (7) and obtain [22]

$$
\mathrm{A}_{m j}^{1} \frac{\partial \mathbf{i}_{m j}}{\partial \varsigma_{i}}+\mathrm{A}_{m j}^{2} \frac{\partial \mathbf{i}_{m j+1}}{\partial \varsigma_{i}}=\frac{\partial \mathrm{b}_{m j}}{\partial \varsigma_{i}}-\frac{\partial \mathrm{A}_{m j}^{1}}{\partial \varsigma_{i}} \mathbf{i}_{m j}-\frac{\partial \mathrm{A}_{m j}^{2}}{\partial \varsigma_{i}} \mathbf{i}_{m j+1}
$$

As for radiance calculations, the linearized layer equations (18) are assembled into a global system of equations for the entire atmosphere. It is worth noticing that the system matrix for derivative calculations coincides with the system matrix for radiance calculations; only the right-hand sides are different. In order to increase the efficiency of the method we compute the partial derivatives with respect to all atmospheric parameters $\varsigma_{i}, i=1, \ldots, N_{\mathrm{p}}$, that is, we solve a system of equations with multiple right-hand sides.

2. In MOME, we linearize the interaction principle equation in (12), and compute the partial derivatives $\partial \mathrm{R}_{m j_{\min }} / \partial \varsigma_{i}$ and $\partial \mathrm{r}_{m j_{\min }} / \partial \varsigma_{i}$ recursively by linearizing the adding formulas (10) and (11). From (10) and (11), it is apparent that the recursion relation involves the partial derivatives $\partial \mathbf{R}_{m j} / \partial \varsigma_{i}$, $\partial \mathbf{T}_{m j} / \partial \varsigma_{i}$ and $\partial \boldsymbol{\Sigma}_{m j}^{ \pm} / \partial \varsigma_{i}$.

To compute $\partial \mathrm{A}_{m j}^{1} / \partial \varsigma_{i}, \partial \mathrm{A}_{m j}^{2} / \partial \varsigma_{i}$ and $\partial \mathrm{b}_{m j} / \partial \varsigma_{i}$ as well as $\partial \mathbf{R}_{m j} / \partial \varsigma_{i}$, $\partial \mathbf{T}_{m j} / \partial \varsigma_{i}$ and $\partial \boldsymbol{\Sigma}_{m j}^{ \pm} / \partial \varsigma_{i}$, we apply the chain rule. In the case of the Padé approximation, the derivative calculations are trivial, but in the case of the eigendecomposition method we face the calculation of the partial derivatives of the inverse of the eigenvector matrix $\mathbf{V}_{m j}^{-1}$ and of the eigenvalues $\lambda_{k}$. This computation step is outlined in Appendix A.

\subsection{Forward-adjoint approach}

The steps of the adjoint radiative transfer approach consist of the formulation of the boundary-value problem for radiative transfer as an operator equation with homogeneous boundary conditions, the derivation of the adjoint radiative transfer operator, and the representation of the measured radiance in terms 
of the solution of the adjoint radiative transfer problem and the forward source function. In our analysis, the forward and the adjoint radiative transfer problem are solved by using DOME.

Essentially, the forward-adjoint approach relies on the following basic result: If the (total) radiance field $I(r, \mathbf{\Omega})$ solves the forward problem

$$
\begin{aligned}
& \mathcal{L} I(r, \boldsymbol{\Omega})=Q(r, \boldsymbol{\Omega}), \\
& I\left(r_{\mathrm{TOA}}, \mathbf{\Omega}^{-}\right)=I\left(r_{\mathrm{s}}, \mathbf{\Omega}^{+}\right)=0,
\end{aligned}
$$

and the radiance field $I^{\dagger}(r, \boldsymbol{\Omega})$ solves the adjoint problem

$$
\begin{aligned}
& \mathcal{L}^{\dagger} I^{\dagger}(r, \boldsymbol{\Omega})=Q^{\dagger}(r, \boldsymbol{\Omega}), \\
& I^{\dagger}\left(r_{\mathrm{TOA}}, \boldsymbol{\Omega}^{+}\right)=I^{\dagger}\left(r_{\mathrm{s}}, \mathbf{\Omega}^{-}\right)=0,
\end{aligned}
$$

then the measured radiance at the top of the atmosphere and in the direction $\boldsymbol{\Omega}_{\mathrm{m}}=\left(\mu_{\mathrm{m}}, \varphi_{\mathrm{m}}\right)$ with $\mu_{\mathrm{m}}>0$, can be computed as

$$
I_{\mathrm{m}}=\left\langle Q^{\dagger}, I\right\rangle=\left\langle I^{\dagger}, Q\right\rangle .
$$

The forward radiative transfer operator $\mathcal{L}$ and the forward source function $Q$ are given by

$$
\begin{aligned}
& \mathcal{L} I(r, \boldsymbol{\Omega})=\mu \frac{\mathrm{d} I}{\mathrm{~d} r}(r, \boldsymbol{\Omega})+\sigma_{\mathrm{ext}}(r) I(r, \boldsymbol{\Omega}) \\
& -\frac{\sigma_{\mathrm{sct}}(r)}{4 \pi} \int_{4 \pi} P\left(r, \boldsymbol{\Omega}, \boldsymbol{\Omega}^{\prime}\right) I\left(r, \boldsymbol{\Omega}^{\prime}\right) \mathrm{d} \Omega^{\prime} \\
& -\frac{A}{\pi} \delta\left(r-r_{\mathrm{s}}\right) H(\mu) \mu \int_{4 \pi} \rho\left(\boldsymbol{\Omega}, \boldsymbol{\Omega}^{\prime}\right) H\left(-\mu^{\prime}\right)\left|\mu^{\prime}\right| I\left(r, \boldsymbol{\Omega}^{\prime}\right) \mathrm{d} \Omega^{\prime}
\end{aligned}
$$

and

$$
Q(r, \boldsymbol{\Omega})=F_{0} \mu_{0} \delta\left(r-r_{\mathrm{TOA}}\right) \delta\left(\mathbf{\Omega}-\mathbf{\Omega}_{0}\right),
$$

respectively, where $\delta$ is the Dirac delta function and $H$ is the Heaviside step function. In this context, the forward problem consisting of the operator equation (19) and the homogeneous boundary conditions (20) is equivalent to the radiative transfer equation (1) with the boundary conditions (3) and (4). The adjoint radiative transfer operator $\mathcal{L}^{\dagger}$ is defined through the Lagrange identity

$$
\left\langle\mathcal{L} I, I^{\dagger}\right\rangle=\left\langle I, \mathcal{L}^{\dagger} I^{\dagger}\right\rangle,
$$

where the scalar product of fields $I_{1}$ and $I_{2}$ is given by

$$
\left\langle I_{1}, I_{2}\right\rangle=\int_{r_{\mathrm{s}}}^{r_{\mathrm{TOA}}} \int_{4 \pi} I_{1}(r, \boldsymbol{\Omega}) I_{2}(r, \mathbf{\Omega}) \mathrm{d} \Omega \mathrm{d} r .
$$

For the homogeneous boundary conditions (20) and (22), the expressions of the adjoint operator $\mathcal{L}^{\dagger}$ and the adjoint source function $Q^{\dagger}$ are

$$
\begin{aligned}
& \mathcal{L}^{\dagger} I^{\dagger}(r, \boldsymbol{\Omega})=-\mu \frac{\mathrm{d} I^{\dagger}}{\mathrm{d} r}(r, \boldsymbol{\Omega})+\sigma_{\mathrm{ext}}(r) I^{\dagger}(r, \boldsymbol{\Omega}) \\
& -\frac{\sigma_{\mathrm{sct}}(r)}{4 \pi} \int_{4 \pi} P\left(r, \boldsymbol{\Omega}^{\prime}, \mathbf{\Omega}\right) I^{\dagger}\left(r, \boldsymbol{\Omega}^{\prime}\right) \mathrm{d} \Omega^{\prime} \\
& \quad-\frac{A}{\pi} \delta\left(r-r_{\mathrm{s}}\right) H(-\mu)|\mu| \int_{4 \pi} \rho\left(\boldsymbol{\Omega}^{\prime}, \mathbf{\Omega}\right) H\left(\mu^{\prime}\right)\left|\mu^{\prime}\right| I^{\dagger}\left(r, \mathbf{\Omega}^{\prime}\right) \mathrm{d} \Omega^{\prime}
\end{aligned}
$$

and

$$
Q^{\dagger}(r, \boldsymbol{\Omega})=\delta\left(r-r_{\mathrm{TOA}}\right) \delta\left(\boldsymbol{\Omega}-\mathbf{\Omega}_{\mathrm{m}}\right),
$$

respectively. The solution of the adjoint radiative transfer problem can be found by using the same solution method as for the forward problem with a modified source function. Actually, it can be shown that the conjugate adjoint radiance $\widehat{I}^{\dagger}$ defined by

$$
\widehat{I}^{\dagger}(r, \mathbf{\Omega})=I^{\dagger}(r,-\mathbf{\Omega})
$$

solves the conjugate adjoint problem

$$
\begin{aligned}
& \widehat{\mathcal{L} \widehat{I}^{\dagger}}(r, \boldsymbol{\Omega})=\widehat{Q}^{\dagger}(r, \boldsymbol{\Omega}), \\
& \widehat{I}^{\dagger}\left(r_{\mathrm{TOA}}, \boldsymbol{\Omega}^{-}\right)=\widehat{I}^{\dagger}\left(r_{\mathrm{s}}, \mathbf{\Omega}^{+}\right)=0,
\end{aligned}
$$

where

$$
\widehat{Q}^{\dagger}(r, \boldsymbol{\Omega})=\widehat{F}_{0} \mu_{\mathrm{m}} \delta\left(r-r_{\mathrm{TOA}}\right) \delta\left(\boldsymbol{\Omega}-\widehat{\mathbf{\Omega}}_{\mathrm{m}}\right)
$$

is the conjugate adjoint source function, $\widehat{\boldsymbol{\Omega}}_{\mathrm{m}}=-\boldsymbol{\Omega}_{\mathrm{m}}=\left(\widehat{\mu}_{\mathrm{m}}, \widehat{\varphi}_{\mathrm{m}}\right)$ is the conjugate adjoint direction $\left(\widehat{\mu}_{\mathrm{m}}=-\mu_{\mathrm{m}}\right.$ and $\left.\widehat{\varphi}_{\mathrm{m}}=\varphi_{\mathrm{m}}+\pi\right)$, and $\widehat{F}_{0}=1 / \mu_{\mathrm{m}}$. The boundary value problems (19)-(20) and (29)-(30) are identical excepting the source functions (25) and (31), which, however, are of similar forms. As a result, in the discrete ordinate method with matrix exponential, the system matrices for the forward and conjugate adjoint problems coincide, and solving a system of equations with two right-hand sides yields the level values of the forward and (conjugate) adjoint radiance fields.

We come now to the derivative calculations. Taking the variation of the measured radiance with respect to variations of atmospheric parameters, and using (21) and (26) yields $\left(\delta Q^{\dagger}=0\right)$

$$
\delta I_{\mathrm{m}}=\left\langle Q^{\dagger}, \delta I\right\rangle=\left\langle I^{\dagger}, \mathcal{L} \delta I\right\rangle
$$

The variation of the forward operator equation (19) gives

$$
\mathcal{L} \delta I=\delta Q-\delta \mathcal{L} I
$$

and, since $\delta Q=0$, we obtain

$$
\delta I_{\mathrm{m}}=-\left\langle I^{\dagger}, \delta \mathcal{L} I\right\rangle .
$$

In the first step of the forward-adjoint approach, we separate the total radiance $I(r, \boldsymbol{\Omega})$ into a diffuse and a direct component $I_{\mathrm{d}}(r, \boldsymbol{\Omega})$ and $I_{\odot}(r, \boldsymbol{\Omega})$, respectively, (cf. (5)), and do the same for the conjugate adjoint total radiance $\widehat{I}^{\dagger}(r, \boldsymbol{\Omega})$, i.e.

$$
\widehat{I^{\dagger}}(r, \boldsymbol{\Omega})=\widehat{I}_{\mathrm{d}}^{\dagger}(r, \mathbf{\Omega})+\widehat{I}_{\odot}^{\dagger}(r, \mathbf{\Omega}),
$$

with

$$
\widehat{I}_{\odot}^{\dagger}(r, \boldsymbol{\Omega})=\widehat{F}_{0} \delta\left(\mathbf{\Omega}-\widehat{\boldsymbol{\Omega}}_{\mathrm{m}}\right) \widehat{T}^{\dagger}(r)
$$

and $\widehat{T}^{\dagger}(r)=\exp \left[-\tau_{\text {ext }}\left(\left|\mathbf{r}-\mathbf{r}_{\mathrm{TOA}}\right|, \widehat{\boldsymbol{\Omega}}_{\mathrm{m}}\right)\right]$. Inserting the resulting expressions in (34) it gives

$$
\frac{\partial I_{\mathrm{m}}}{\partial \varsigma_{i}}=T_{1}+T_{2}
$$




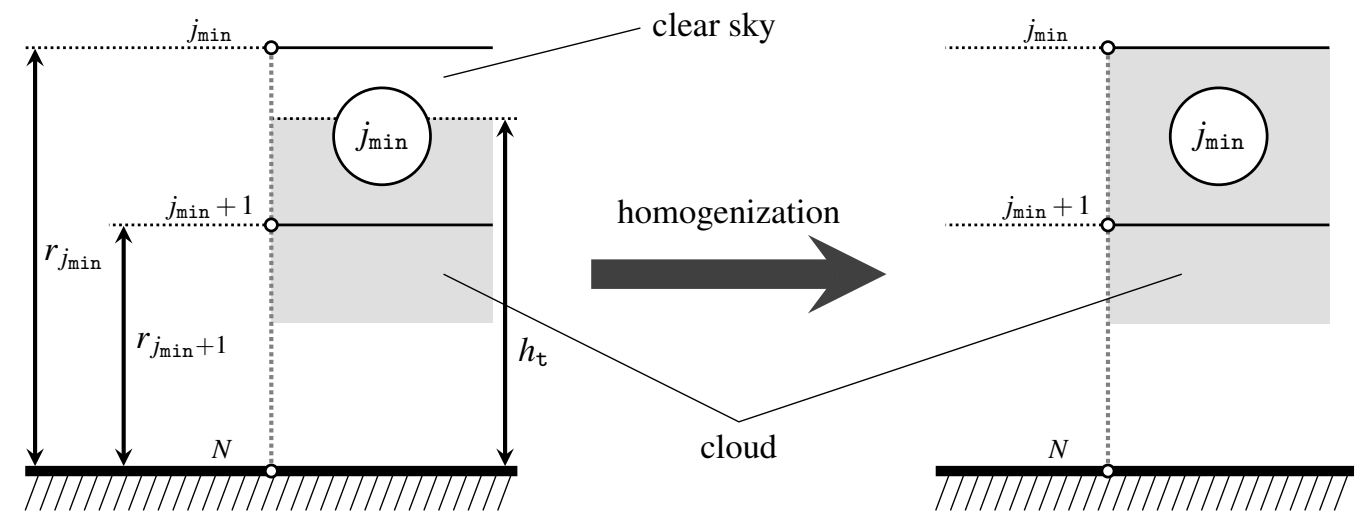

Figure 1: Homogenization of the atmospheric layer $j_{\text {min }}$ containing the cloud top height $h_{\mathrm{t}}$. The layer $j_{\text {min }}$, with the boundary levels $j_{\text {min }}$ and $j_{\text {min }}+1$, is indicated by a circle.

where the first term $T_{1}$ involves integrals of the conjugate adjoint diffuse radiance $\widehat{I_{\mathrm{d}}^{\dagger}}$,

$$
\begin{aligned}
T_{1} & =-\iint_{r_{\mathrm{s}}}^{r_{\text {TOA }}} \frac{\partial \sigma_{\mathrm{ext}}}{\partial \varsigma_{i}}(r) \widehat{I}_{\mathrm{d}}^{\dagger}(r,-\boldsymbol{\Omega}) I_{\mathrm{d}}(r, \boldsymbol{\Omega}) \mathrm{d} r \mathrm{~d} \Omega \\
& -F_{0} \int_{r_{\mathrm{s}}}^{r_{\text {TOA }}} \frac{\partial \sigma_{\mathrm{ext}}}{\partial \varsigma_{i}}(r) \widehat{I}_{\mathrm{d}}^{\dagger}\left(r,-\boldsymbol{\Omega}_{0}\right) T(r) \mathrm{d} r \\
& +\frac{1}{4 \pi} \int_{r_{\mathrm{s}}}^{r_{\text {TOA }}} \widehat{I}_{\mathrm{d}}^{\dagger}(r,-\mathbf{\Omega}) \mathrm{d} r \mathrm{~d} \Omega \int_{4 \pi} \frac{\partial}{\partial \varsigma_{i}}\left[\sigma_{\mathrm{sct}}(r) P\left(r, \boldsymbol{\Omega}, \boldsymbol{\Omega}^{\prime}\right)\right] I_{\mathrm{d}}\left(r, \mathbf{\Omega}^{\prime}\right) \mathrm{d} \Omega^{\prime} \\
& +\frac{F_{0}}{4 \pi} \int_{r_{\mathrm{s}}}^{r_{\text {TOA }}} \int_{4 \pi} \frac{\partial}{\partial \varsigma_{i}}\left[\sigma_{\mathrm{sct}}(r) P\left(r, \boldsymbol{\Omega}, \mathbf{\Omega}_{0}\right)\right] \widehat{I}_{\mathrm{d}}^{\dagger}(r,-\boldsymbol{\Omega}) T(r) \mathrm{d} r \mathrm{~d} \Omega,
\end{aligned}
$$

while the second term $T_{2}$ involves integrals of the (conjugate) adjoint transmission $\widehat{T}^{\dagger}$,

$$
\begin{aligned}
T_{2} & =-\widehat{F}_{0} \int_{r_{\mathrm{s}}}^{r_{\mathrm{TOA}}} \frac{\partial \sigma_{\mathrm{ext}}}{\partial \varsigma_{i}}(r) I_{\mathrm{d}}\left(r, \mathbf{\Omega}_{\mathrm{m}}\right) \widehat{T}^{\dagger}(r) \mathrm{d} r \\
& +\frac{\widehat{F}_{0}}{4 \pi} \int_{r_{\mathrm{s}}}^{r_{\mathrm{TOA}}} \widehat{T}^{\dagger}(r) \mathrm{d} r \int_{4 \pi} \frac{\partial}{\partial \varsigma_{i}}\left[\sigma_{\mathrm{sct}}(r) P\left(r, \mathbf{\Omega}_{\mathrm{m}}, \mathbf{\Omega}^{\prime}\right)\right] I_{\mathrm{d}}\left(r, \mathbf{\Omega}^{\prime}\right) \mathrm{d} \Omega^{\prime} \\
& +\frac{\widehat{F}_{0} F_{0}}{4 \pi} \int_{r_{\mathrm{s}}}^{r_{\mathrm{TOA}}} \frac{\partial}{\partial \varsigma_{i}}\left[\sigma_{\mathrm{sct}}(r) P\left(r, \mathbf{\Omega}_{\mathrm{m}}, \mathbf{\Omega}_{0}\right)\right] T(r) \widehat{T}^{\dagger}(r) \mathrm{d} r
\end{aligned}
$$

As a next step, the integration over the azimuthal angle in (37) and (38) is performed. This computation step together with the integration over the radial coordinate is described in Appendix B.

\subsection{Derivatives with respect to cloud geometrical parameters}

We must be cautious when computing the derivatives with respect to the cloud geometrical parameters (cloud top height $h_{\mathrm{t}}$ and cloud bottom height $h_{\mathrm{b}}$ ). In fact, this process is intimately connected with the discretization of the atmosphere containing an homogeneous cloud. We have two options:
1. the atmosphere above, below and in the cloud is discretized, so that the cloud top and bottom heights are atmospheric levels;

2. the atmosphere is discretized into a fixed grid, and the layers containing the cloud top and bottom heights are homogenized.

The first discretization method, in which the atmosphere is discretized each time when a new cloud position is considered, can be used in conjunction with the forward-adjoint approach and is described in Appendix C. The second discretization method, in which a fixed altitude grid is used, can be applied for both the linearized forward and forward-adjoint approaches and is summarized below.

Let the optical properties of the homogeneous cloud be described by the cloud extinction coefficient $\sigma_{\text {ext }}^{\mathrm{c}}$ and the expansion coefficients $\xi_{n}^{\mathrm{c}}$. Assume that the cloud top height $h_{\mathrm{t}}$ lies between the levels $r_{j_{\min }+1}$ and $r_{j_{\min }}$, i.e. $r_{j_{\min }+1}<h_{\mathrm{t}}<r_{j_{\min }}$ as shown in Fig. 1. In layer $j_{\text {min }}$ containing the cloud top height $h_{\mathrm{t}}$, we perform an homogenization which consists of the computation of the optical parameters $\sigma_{\text {ext } j_{\min }}$ and $\xi_{n j_{\min }}$ by using

$$
\begin{aligned}
\sigma_{\text {ext } j_{\min }} & =\sigma_{\text {ext } j_{\min }}^{0}+w\left(h_{\mathrm{t}}\right) \sigma_{\mathrm{ext}}^{\mathrm{c}}, \\
\xi_{n j_{\min }} & =\xi_{n j_{\min }}^{0}+w\left(h_{\mathrm{t}}\right) \xi_{n}^{\mathrm{c}},
\end{aligned}
$$

where the superscript " 0 " refers to the clear sky atmosphere, and the weighting factor $w$ is given by

$$
w\left(h_{\mathrm{t}}\right)=\frac{h_{\mathrm{t}}-r_{j_{\min }+1}}{\Delta r_{j}} .
$$

By this procedure, $\sigma_{\text {ext } j_{\min }}$ and $\xi_{n j_{\min }}$ are functions of $h_{\mathrm{t}}$, i.e.

$$
\sigma_{\text {ext } j_{\min }}=\sigma_{\text {ext } j_{\min }}\left(\sigma_{\text {ext }}^{\mathrm{c}}, h_{\mathrm{t}}\right) \quad \text { and } \quad \xi_{n j_{\min }}=\xi_{n j_{\min }}\left(\sigma_{\mathrm{ext}}^{\mathrm{c}}, h_{\mathrm{t}}\right) \text {, }
$$

so that the partial derivatives $\partial \sigma_{\text {ext } j_{\min }} / \partial h_{\mathrm{t}}$ and $\partial \xi_{n j_{\min }} / \partial h_{\mathrm{t}}$ can be readily computed. The derivatives with respect to the cloud bottom height $h_{\mathrm{b}}$ are computed in an analogous manner. Note that, for an accurate homogenization, the discretization step $\Delta r_{j}$ should not be too large. 


\subsection{PCA-based methods for derivative calculations}

As for radiance calculations, acceleration techniques can be used to increase the computation speed. These include the Padé approximation, the delta-M scaling and TMS correction methods, the telescoping technique, the method of false discrete ordinate, the correlated $k$-distribution method, and the PCA technique. For derivative calculations, two PCA-based methods can be designed. To explain these methods, we give a short overview of dimensionality reduction techniques for optical parameters [9].

An approximate model for computing the radiance $I$ at the wavelength $\lambda$ reads as

$$
\ln \frac{I(\lambda)}{I_{\mathrm{a}}(\lambda)}=f_{\mathrm{I}}(\lambda)
$$

where $I_{\mathrm{a}}$ is the radiance computed by an approximate radiative transfer model, and $f_{\mathrm{I}}$ is a correction factor.

The optical property dimensionality reduction is performed on the layer values of the gas absorption coefficient $\ln \sigma_{\text {abs }}^{\text {gas }}(\lambda)$ and the molecular scattering coefficient $\ln \sigma_{\text {sct }}^{\text {mol }}(\lambda)$. For each $\lambda_{k}, k=1, \ldots N_{\lambda}$, where $N_{\lambda}$ is the number of discrete wavelengths, we define an $\mathcal{N}$-dimensional vector (the abbreviation "not" stands for notation)

$$
\mathbf{x}\left(\lambda_{k}\right) \stackrel{\text { not }}{=} \mathbf{x}_{k}=\left[\ln \sigma_{\mathrm{abs} j}^{\mathrm{gas}}\left(\lambda_{k}\right) ; \ln \sigma_{\mathrm{sct} j}^{\mathrm{mol}}\left(\lambda_{k}\right)\right]^{\top}, j=1, \ldots, N-1,
$$

with $\mathcal{N}=2 N-2$, so that the wavelength variability of the optical parameters is contained in $\mathbf{x}_{k}$. High-dimensional real data often lie on or near a lower-dimensional manifold. The fundamental issues in dimensionality reduction are the modeling of the geometry structure of the manifold, and the design of an appropriate embedding for data projection. For the $\mathcal{N}$-dimensional data set $\left\{\mathbf{x}_{k}\right\}_{k=1}^{N_{\lambda}}$, where $\mathbf{x}_{k} \in \mathbb{R}^{\mathcal{N}}$, let $\overline{\mathbf{x}}=\left(1 / N_{\lambda}\right) \sum_{k=1}^{N_{\lambda}} \mathbf{x}_{k}$ be the sample mean of the data. The goal of a linear embedding method is to find an $\mathcal{M}$-dimensional subspace $(\mathcal{M}<\mathcal{N})$ spanned by a set of linear independent vectors $\left\{\mathbf{a}_{l}\right\}_{l=1}^{\mathcal{M}}$, such that the centered data $\mathbf{x}_{k}-\overline{\mathbf{x}}$ lie mainly on this subspace (manifold), i.e. $\mathbf{x}_{k} \approx \overline{\mathbf{x}}+\sum_{l=1}^{\mathcal{M}} y_{k l} \mathbf{a}_{l}=\overline{\mathbf{x}}+\mathbf{A} \mathbf{y}_{k}, k=1, \ldots, N_{\lambda}$. Here, $\mathbf{A} \stackrel{\text { not }}{=}\left[\mathbf{a}_{l}\right]_{l=1}^{\mathcal{M}}$ is an $\mathcal{N} \times \mathcal{M}$ matrix comprising the column vectors $\mathbf{a}_{l}$, and $y_{k l}$ is the $l$ th component of the vector of parameters $\mathbf{y}_{k} \in \mathbb{R}^{\mathcal{M}}$. The vector of parameters $\mathbf{y}_{k}$ is given by the forward mapping from the high-dimensional space to the low-dimensional space, i.e. $\mathbf{y}_{k}=\mathbf{A}^{\dagger}\left(\mathbf{x}_{k}-\overline{\mathbf{x}}\right)$, where $\mathbf{A}^{\dagger}=\left(\mathbf{A}^{\top} \mathbf{A}\right)^{-1} \mathbf{A}^{\top}$ is the pseudoinverse of $\mathbf{A}$. Now, let $f\left(\mathbf{x}_{k}\right)$ be a scalar function, which by assumption is not too nonlinear in $\mathbf{x}_{k}$. Setting

$$
\Delta \mathbf{x}_{k}=\sum_{l=1}^{\mathcal{M}} y_{k l} \mathbf{a}_{l},
$$

we approximate $f\left(\mathbf{x}_{k}\right)$ by a second-order Taylor expansion, expressed in finite-difference form by

$$
\begin{aligned}
f\left(\mathbf{x}_{k}\right) & \approx f(\overline{\mathbf{x}})+\frac{1}{2} \sum_{l=1}^{\mathcal{M}}\left[f\left(\overline{\mathbf{x}}+\mathbf{a}_{l}\right)-f\left(\overline{\mathbf{x}}-\mathbf{a}_{l}\right)\right] y_{k l} \\
& +\frac{1}{2} \sum_{l=1}^{\mathcal{M}}\left[f\left(\overline{\mathbf{x}}+\mathbf{a}_{l}\right)-2 f(\overline{\mathbf{x}})+f\left(\overline{\mathbf{x}}-\mathbf{a}_{l}\right)\right] y_{k l}^{2} .
\end{aligned}
$$

To compute the radiance correction factor we identify

$$
f\left(\mathbf{x}_{k}\right)=f_{\mathrm{I}}\left(\lambda_{k}\right)=\ln \left[I\left(\lambda_{k}\right) / I_{\mathrm{a}}\left(\lambda_{k}\right)\right],
$$

and from (45) it is apparent that the computation of the correction factor requires $2 \mathcal{M}+1$ calls of the exact and approximate models. As a result and taking into account that $\mathcal{M} \ll N_{\lambda}$, we are led to a substantial reduction of the computational time. A short remark is though needed. PCA produces a global linear model of the data and is appropriate when the manifold is embedded linearly or almost linearly in the data space. The method preserves only the global structure of the data, and may fail to preserve the local structure if the data lies on a nonlinear manifold. In contrast, the linear embedding methods presented in [9] optimally preserve local neighbourhood information (the local structure of the data) in a certain sense. In fact, the dimensionality reduction approach used in $[6,7]$ is also a local linear model, which combines PCA with the clustering of the data space. In general, a local model implementation of PCA involves a twostep procedure: (1) a clustering of the data space into disjoint regions by using, for instance, the Lloyd algorithm with Euclidean distances as the distortion function, and (2) the estimation of the linear mappings within each region by PCA. Then, each region (bin of wavelengths) is characterized by its own orthogonal basis, and so, by its own set of correction factors. If $P$ is the number of the disjoint regions, then $(2 \mathcal{M}+1) P$ calls of the exact and the approximate models are required to compute all correction factors.

Two methods can be used for computing the derivatives with respect to the atmospheric parameter $\varsigma_{i}$.

1. The first method is similar to (46), and uses the identification

$$
f\left(\mathbf{x}_{k}\right)=f_{\varsigma_{i}}\left(\lambda_{k}\right)=\ln \left[\frac{\partial I}{\partial \varsigma_{i}}\left(\lambda_{k}\right) / \frac{\partial I_{\mathrm{a}}}{\partial \varsigma_{i}}\left(\lambda_{k}\right)\right] .
$$

2. The second method is based on the linearization of the restoration equation (45) for the radiance correction factor $f_{\mathrm{I}}$, and involves the computation of the derivatives $\partial y_{k l} / \partial \varsigma_{i}$ [8]. In our framework, this technique can be summarized as follows. Express the restoration equation as

$$
I\left(\lambda_{k}\right)=I_{\mathrm{a}}\left(\lambda_{k}\right) \mathrm{e}^{f_{\mathrm{I}}\left(\lambda_{k}\right)}
$$

and take the derivative to obtain

$$
\frac{\partial I}{\partial \varsigma_{i}}\left(\lambda_{k}\right)=\frac{\partial I_{\mathrm{a}}}{\partial \varsigma_{i}}\left(\lambda_{k}\right) \mathrm{e}^{f_{\mathrm{I}}\left(\lambda_{k}\right)}+I_{\mathrm{a}}\left(\lambda_{k}\right) \mathrm{e}^{f_{\mathrm{I}}\left(\lambda_{k}\right)} \frac{\partial f_{\mathrm{I}}}{\partial \varsigma_{i}}\left(\lambda_{k}\right)
$$

with

$$
\begin{aligned}
& \frac{\partial f_{\mathrm{I}}}{\partial \varsigma_{i}}\left(\lambda_{k}\right) \equiv \frac{\partial f}{\partial \varsigma_{i}}\left(\mathbf{x}_{k}\right) \\
& \quad \frac{\partial f}{\partial \varsigma_{i}}(\overline{\mathbf{x}})+\frac{1}{2} \sum_{l=1}^{\mathcal{M}}\left[\frac{\partial f}{\partial \varsigma_{i}}\left(\overline{\mathbf{x}}+\mathbf{a}_{l}\right)-\frac{\partial f}{\partial \varsigma_{i}}\left(\overline{\mathbf{x}}-\mathbf{a}_{l}\right)\right] y_{k l} \\
& \quad+\frac{1}{2} \sum_{l=1}^{\mathcal{M}}\left[f\left(\overline{\mathbf{x}}+\mathbf{a}_{l}\right)-f\left(\overline{\mathbf{x}}-\mathbf{a}_{l}\right)\right] \frac{\partial y_{k l}}{\partial \varsigma_{i}} \\
& +\frac{1}{2} \sum_{l=1}^{\mathcal{M}}\left[\frac{\partial f}{\partial \varsigma_{i}}\left(\overline{\mathbf{x}}+\mathbf{a}_{l}\right)-2 \frac{\partial f}{\partial \varsigma_{i}}(\overline{\mathbf{x}})+\frac{\partial f}{\partial \varsigma_{i}}\left(\overline{\mathbf{x}}-\mathbf{a}_{l}\right)\right] y_{k l}^{2} \\
& \quad+\sum_{l=1}^{\mathcal{M}}\left[f\left(\overline{\mathbf{x}}+\mathbf{a}_{l}\right)-2 f(\overline{\mathbf{x}})+f\left(\overline{\mathbf{x}}-\mathbf{a}_{l}\right)\right] y_{k l} \frac{\partial y_{k l}}{\partial \varsigma_{i}}
\end{aligned}
$$


In general, from a vector $\mathbf{x}$ (which can be identified with $\overline{\mathbf{x}}$ or $\overline{\mathbf{x}} \pm \mathbf{a}_{l}$ ) we extract $\left\{\sigma_{\text {abs } j}^{\text {gas }}\right\}_{j=1}^{N-1}$ and $\left\{\sigma_{\text {sct } j}^{\text {mol }}\right\}_{j=1}^{N-1}$ according to the representation $\mathbf{x}=\left[\ln \sigma_{\text {abs } j}^{\text {gas }} ; \ln \sigma_{\text {sct } j}^{\text {mol }}\right]^{\top}$ and, for these optical parameters, we compute the exact and approximate radiances $I(\mathbf{x})$ and $I_{\mathrm{a}}(\mathbf{x})$, respectively. Then, we have

$$
\frac{\partial f}{\partial \varsigma_{i}}(\mathbf{x})=\frac{1}{I(\mathbf{x}) I_{\mathrm{a}}(\mathbf{x})}\left[\frac{\partial I}{\partial \varsigma_{i}}(\mathbf{x}) I_{\mathrm{a}}(\mathbf{x})-\frac{\partial I_{\mathrm{a}}}{\partial \varsigma_{i}}(\mathbf{x}) I(\mathbf{x})\right],
$$

and what is left is the computation of $\partial y_{k l} / \partial \varsigma_{i}$. For doing this, we need to specify the mapping from the highdimensional space to the low-dimensional space. Let PCA be chosen as the dimensionality reduction technique, and let us assume that all centered data $\mathbf{x}_{k}-\overline{\mathbf{x}}, k=1, \ldots, N_{\lambda}$, are stacked into the columns of an $\mathcal{N} \times N_{\lambda}$ matrix $\mathbf{X}$, i.e. $\mathbf{X}=\left[\mathbf{x}_{k}-\overline{\mathbf{x}}\right]_{k=1}^{N_{\lambda}}$. Essentially, PCA performs a dimensionality reduction by projecting the original $\mathcal{N}$-dimensional data on the $\mathcal{M}$-dimensional subspace spanned by the dominant singular vectors of the data's covariance matrix. Therefore, assuming the singular value decomposition $\mathbf{C}_{\mathbf{x}}=\left(1 / N_{\lambda}\right) \mathbf{X} \mathbf{X}^{\boldsymbol{\top}}=\mathbf{U} \boldsymbol{\Sigma} \mathbf{U}^{\top}$, where $\boldsymbol{\Sigma} \stackrel{\text { not }}{=} \operatorname{diag}\left[\sigma_{l}\right]_{l=1}^{\mathcal{N}}$ is the $\mathcal{N} \times \mathcal{N}$ diagonal matrix of the singular values appearing in decreasing order $\sigma_{1}>\sigma_{2}>\cdots>\sigma_{\mathcal{N}}>0$, and $\mathbf{U}=\left[\mathbf{u}_{l}\right]_{l=1}^{\mathcal{N}}$ is the $\mathcal{N} \times \mathcal{N}$ orthogonal (or orthonormal) matrix of the singular vectors $\mathbf{u}_{l}$, we take $\mathbf{A}=\mathbf{U}_{\mathcal{M}}=\left[\mathbf{u}_{l}\right]_{l=1}^{\mathcal{M}}$, yielding $\mathbf{A}^{\dagger}=\mathbf{U}_{\mathcal{M}}^{\top}$. As in $[6,7]$, we consider the scaled orthogonal vectors $\overline{\mathbf{u}}_{l}=\sigma_{l} \mathbf{u}_{l}$, in which case we have $\mathbf{A}=\overline{\mathbf{U}}_{\mathcal{M}}=\mathbf{U}_{\mathcal{M}} \boldsymbol{\Sigma}_{\mathcal{M}}$ and $\mathbf{A}^{\dagger}=\overline{\mathbf{U}}_{\mathcal{M}}^{\dagger}=\boldsymbol{\Sigma}_{\mathcal{M}}^{-1} \mathbf{U}_{\mathcal{M}}^{\top}$, with $\boldsymbol{\Sigma}_{\mathcal{M}}=\operatorname{diag}\left[\sigma_{l}\right]_{l=1}^{\mathcal{M}}$. We then get

$$
y_{k l}=\frac{1}{\sigma_{l}} \mathbf{u}_{l}^{\top}\left(\mathbf{x}_{k}-\overline{\mathbf{x}}\right),
$$

and, further,

$$
\begin{aligned}
\frac{\partial y_{k l}}{\partial \varsigma_{i}} & =-\frac{1}{\sigma_{l}^{2}} \mathbf{u}_{l}^{\top}\left(\mathbf{x}_{k}-\overline{\mathbf{x}}\right) \frac{\partial \sigma_{l}}{\partial \varsigma_{i}}+\frac{1}{\sigma_{l}}\left(\frac{\partial \mathbf{u}_{l}}{\partial \varsigma_{i}}\right)^{\top}\left(\mathbf{x}_{k}-\overline{\mathbf{x}}\right) \\
& +\frac{1}{\sigma_{l}} \mathbf{u}_{l}^{\top}\left(\frac{\partial \mathbf{x}_{k}}{\partial \varsigma_{i}}-\frac{\partial \overline{\mathbf{x}}}{\partial \varsigma_{i}}\right) .
\end{aligned}
$$

To compute $\partial y_{k l} / \partial \varsigma_{i}$ we need $\partial \sigma_{l} / \partial \varsigma_{i}$ and $\partial \mathbf{u}_{l} / \partial \varsigma_{i}$. As $\mathbf{C}_{\mathbf{x}} \mathbf{u}_{l}=\sigma_{l} \mathbf{u}_{l}$ and $\mathbf{u}_{l}^{\top} \mathbf{u}_{l}=1$, these quantities can be computed by solving the $(\mathcal{N}+1) \times(\mathcal{N}+1)$ system of equations (as in Appendix A)

$$
\left[\begin{array}{cc}
\mathbf{u}_{l} & \sigma_{l} \mathbf{I}_{\mathcal{N}}-\mathbf{C}_{\mathbf{x}} \\
0 & \mathbf{u}_{l}^{\top}
\end{array}\right]\left[\begin{array}{c}
\frac{\partial \sigma_{l}}{\partial \varsigma_{i}} \\
\frac{\partial \mathbf{u}_{l}}{\partial \varsigma_{i}}
\end{array}\right]=\left[\begin{array}{c}
\frac{\partial \mathbf{C}_{\mathbf{x}}}{\partial \varsigma_{i}} \mathbf{u}_{l} \\
0
\end{array}\right] .
$$

The disadvantage of the first method is that the second-order Taylor approximation should be valid for both $f_{\mathrm{I}}\left(\lambda_{k}\right)$ and $f_{S_{i}}\left(\lambda_{k}\right)$, while the disadvantage of the second method lies in an increase of the computation time. However, in the second method, if $\varsigma_{i}$ stands for the cloud optical thickness or the cloud top height, then $\mathbf{x}_{k}$ does not depend on $\varsigma_{i}$, and so, $\partial y_{k l} / \partial \varsigma_{i}=0$. Consequently, the system of equations (54) needs not to be solved, and the computation time of the second method is comparable to that of the first method.

In [4], the correlated $k$-distribution method has been combined with the PCA technique to speed-up the radiance calculations. The same technique is used here for the derivative calculations.

\section{Numerical simulations}

In this section, we analyze the accuracy and efficiency of the linearized radiative transfer models in computing the derivatives of the measured radiance with respect to the cloud optical thickness $\tau_{\mathrm{c}}$ and the cloud top height $h_{\mathrm{t}}$. The models to be analyzed are the Linearized Discrete Ordinate method with Matrix Exponential (LDOME), the Linearized Matrix Operator method with Matrix Exponential (LMOME), and the ForwardAdjoint approach using the Discrete Ordinate method with Matrix Exponential (FADOME). The derivatives with respect to the cloud geometrical parameters are computed by homogenizing the layers containing the cloud top and bottom heights. The simulations were performed for channel 9 of the EPIC instrument, which is an oxygen A-band absorption channel at $764 \mathrm{~nm}$ with a bandwidth of $1.0 \mathrm{~nm}$. As in [4], we consider a watercloud model with a Gamma size distribution

$$
P(a) \propto a^{\alpha} \exp \left[-\alpha\left(\frac{a}{a_{\text {mod }}}\right)\right]
$$

of parameters $a_{\mathrm{mod}}=8 \mu \mathrm{m}$ and $\alpha=6$. The droplet size ranges between 0.02 and $50.0 \mu \mathrm{m}$, and the cloud geometrical thickness is $\Delta h=h_{\mathrm{t}}-h_{\mathrm{b}}=1.5 \mathrm{~km}$. The atmosphere is discretized with a step of $0.5 \mathrm{~km}$ between 0 and $16 \mathrm{~km}$, a step of $2 \mathrm{~km}$ between 16 and $20 \mathrm{~km}$, a step of $5 \mathrm{~km}$ between 20 and $30 \mathrm{~km}$, and, finally, a step of $10 \mathrm{~km}$ between 30 and $50 \mathrm{~km}$. The ground surface is Lambertian with albedo $A=0.2$, the solar and viewing zenith angles are $\theta_{\mathrm{m}}=\theta_{0}=30^{\circ}$, and the relative azimuthal angle is $\Delta \varphi=\varphi_{\mathrm{m}}-\varphi_{0}=176^{\circ}$. In [4], it was found that a compromise between an accurate description of the scattering in the backward direction and computation time can be reached for a number of discrete ordinates $M$ in the range $24 \leq M \leq 32$. For this reason, we fix the number of discrete ordinates to 32 . The radiances are solar-flux normalized, and the delta-M scaling together with the TMS correction is used. The simulations were performed on a server Intel(R) Xeon(R) CPU E5-2695 v3 @ 2.30 GHz using up to 56 threads.

In Fig. 2, we plot the derivatives with respect to $\tau_{\mathrm{c}}$ for $h_{\mathrm{t}}=4 \mathrm{~km}$, and with respect to $h_{\mathrm{t}}$ for $\tau_{\mathrm{c}}=5$. The results are computed by using line-by-line (LBL) calculations for the oxygen absorption cross sections as in [31,32]. The agreement between LDOME and LMOME is perfect. The results corresponding to FADOME show small deviations from LDOME results; in general, the relative errors are larger when the derivative values are smaller, but they are below $10^{-4}$ for $\partial I_{\mathrm{m}} / \partial \tau_{\mathrm{c}}$ and below $10^{-3}$ for $\partial I_{\mathrm{m}} / \partial h_{\mathrm{t}}$. Note that, in atmospheric remote sensing, due to the general nature of the Gauss-Newton iterative method commonly used to solve the nonlinear inverse problem, the accuracies of the weighting functions up to a few percent do not deteriorate the convergence rate of the solution, and the application of the adjoint approach is not critical. Therefore, an accuracy better than $10^{-3}$ in the derivatives when using FADOME in conjunction with LBL calculations is more than satisfactory.

In Fig. 3, we show the relative errors when LBL calculations are replaced with the correlated $k$-distribution method, the PCA technique, and the correlated $k$-distribution plus PCA method. 

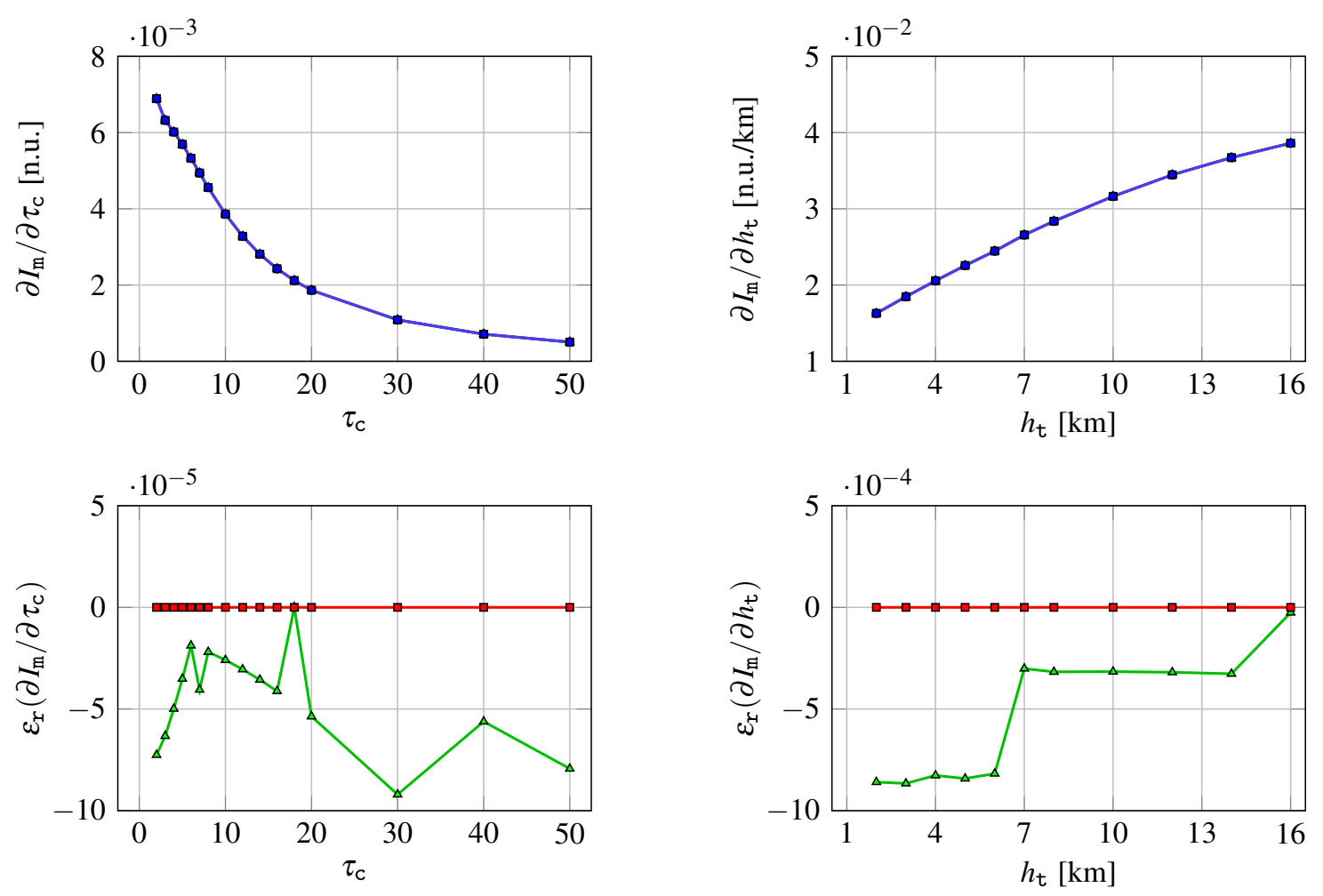

$\rightarrow$ LDOME $\rightarrow$ LMOME $\rightarrow$ FADOME

Figure 2: Upper panels: Radiance derivatives (n.u. denotes Sun-normalized units) with respect to $\tau_{\mathrm{c}}$ for $h_{\mathrm{t}}=4 \mathrm{~km}$ (upper-left panel) and with respect to $h_{\mathrm{t}}$ for $\tau_{\mathrm{c}}=5$ (upper-right panel). Lower panels: Relative errors in the derivatives when using the linearized DOME approach as a reference.

PCA is used with two principal components and one wavelength bin, while the method based on the linearization of the restoration equation (45) is used for derivative calculations. The general observation is that the relative errors in $\partial I_{\mathrm{m}} / \partial \tau_{\mathrm{c}}$ are smaller than $5 \cdot 10^{-3}$, and that the relative errors in $\partial I_{\mathrm{m}} / \partial h_{\mathrm{t}}$ are smaller than $10^{-2}$. Indeed, the relative errors of the correlated $k$-distribution plus PCA method are smaller than $4 \cdot 10^{-3}$ for $\partial I_{\mathrm{m}} / \partial \tau_{\mathrm{c}}$ in the range $2 \leq \tau_{\mathrm{c}} \leq 50$, and smaller than $7 \cdot 10^{-3}$ for $\partial I_{\mathrm{m}} / \partial h_{\mathrm{t}}$ in the range $2 \leq h_{\mathrm{t}} \leq 16 \mathrm{~km}$.

In Table 1 and Table 2, we show the computation times (i.e. the accumulated time over all threads) for the simulations with the linearized radiative transfer models and various acceleration techniques. It is apparent that FADOME is the fastest method, followed by LDOME, equally whether LBL calculations or acceleration techniques are used. The best time performance is obtained when FADOME is combined with the correlated $k$-distribution plus PCA method. In this case, and taking into account that the results in Table 1 correspond to 16 values of $\tau_{\mathrm{c}}$, and the results in Table 2 correspond to 11 values of $h_{\mathrm{t}}$, the average time to simultaneously compute $\partial I_{\mathrm{m}} / \partial \tau_{\mathrm{c}}$ and $\partial I_{\mathrm{m}} / \partial h_{\mathrm{t}}$ at $\left(\tau_{\mathrm{c}}, h_{\mathrm{t}}\right)$ is $13 \mathrm{~s}$.

\section{Conclusions}

Several linearized radiative transfer models have been analyzed with respect to their applicability to the retrieval of cloud parameters from EPIC measurements. The models under consideration are the linearized discrete ordinate method with matrix exponential, the linearized matrix operator method with matrix exponential, and a forward-adjoint approach based on the discrete ordinate method with matrix exponential.

The numerical simulations show that these three models provide similar results when computing the derivatives with respect to the cloud optical thickness and the cloud top height. Furthermore, the forward-adjoint approach based on the discrete ordinate method with matrix exponential, and using the correlated $k$-distribution method in conjunction with the PCA technique, is an accurate and efficient tool for the offline retrieval of cloud optical thickness and cloud top height from EPIC measurements, with a speed-up factor of 2 when compared to the conventional linearization approaches. This forward-adjoint radiative transfer model can be combined with a linearized Mie or T-matrix code [33] to retrieve microphysical properties of clouds and aerosols.

\section{Acknowledgements}

This work was funded by the programme DLR/DAAD Research Fellowships 2015 (57186656), with reference numbers 91613528 and 91627488 , organized by the German Academic Exchange Service (DAAD) and the German Aerospace Center (DLR). 

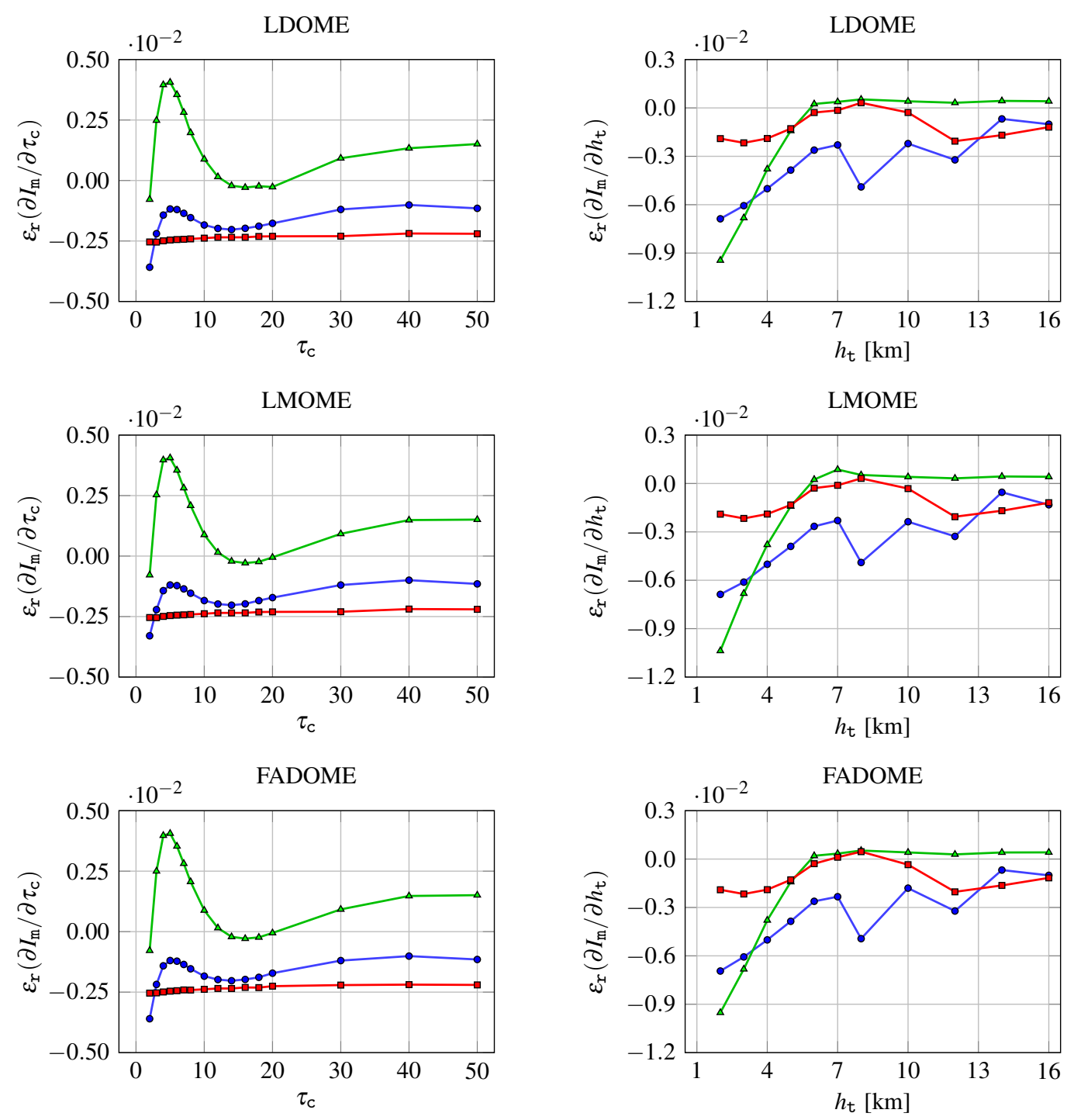

$\rightarrow-$ corr. $k$-distribution $\rightarrow$ PCA technique $\rightarrow-$ corr. $k$-distribution plus PCA

Figure 3: Relative errors in $\partial I_{\mathrm{m}} / \partial \tau_{\mathrm{c}}$ (left panels) and $\partial I_{\mathrm{m}} / \partial h_{\mathrm{t}}$ (right panels) when comparing LBL-based simulations with those for correlated $k$-distribution, PCA technique, and correlated $k$-distribution plus PCA.

Table 1: Computation times in minutes to simulate the derivatives with respect to $\tau_{\mathrm{c}}$ for different models (LDOME, LMOME, FADOME) and acceleration techniques. The speed-up factor corresponds to the ratio between LDOME and FADOME.

\begin{tabular}{lrrrrr}
\hline \multirow{2}{*}{ Acceleration techniques } & \multicolumn{3}{c}{ Linearized models } & \multirow{2}{*}{$\begin{array}{c}\text { Speed-up } \\
\text { factor }\end{array}$} \\
\cline { 2 - 5 } & LDOME & LMOME & FADOME & factonn \\
\hline LBL calculation & 3625 & 4602 & 1804 & 2.0 \\
\hline Correlated $k$-distribution & 678 & 869 & 335 & 2.0 \\
\hline PCA technique & 8 & 9.5 & 6 & 1.3 \\
\hline Corr. $k$-distribution plus PCA technique & 6 & 7.5 & 3.5 & 1.7 \\
\hline
\end{tabular}

Table 2: Computation times in minutes to simulate the derivatives with respect to $h_{\mathrm{t}}$ for different models (LDOME, LMOME, FADOME) and acceleration techniques. The speed-up factor corresponds to the ratio between LDOME and FADOME.

\begin{tabular}{lccccc}
\hline \multirow{2}{*}{ Acceleration techniques } & \multicolumn{3}{c}{ Linearized models } & \multirow{2}{*}{$\begin{array}{c}\text { Speed-up } \\
\text { factor }\end{array}$} \\
\cline { 2 - 5 } & LDOME & LMOME & FADOME & fadonn \\
\hline LBL calculation & 2451 & 3079 & 1240 & 2.0 \\
\hline Correlated $k$-distribution & 456 & 574 & 224 & 2.0 \\
\hline PCA technique & 5.5 & 6.5 & 4.5 & 1.2 \\
\hline Corr. $k$-distribution plus PCA technique & 4 & 5 & 2.5 & 1.6 \\
\hline
\end{tabular}




\section{Appendix A}

In this appendix we compute the partial derivatives of the inverse of the eigenvector matrix $\mathbf{V}_{m j}^{-1}$ and the eigenvalues $\lambda_{k}$.

The steps for computing an eigensystem of the matrix $\mathbf{A}_{m j}$ can be summarized as follows:

1. Compute

$$
\mathcal{A}_{+}=\mathbf{A}_{-} \mathbf{A}_{+},
$$

where $\mathbf{A}_{+}=\mathbf{A}_{m j}^{11}+\mathbf{A}_{m j}^{12}$ and $\mathbf{A}_{-}=\mathbf{A}_{m j}^{11}-\mathbf{A}_{m j}^{12}$, and determine an eigensystem $\left\{\mu_{k}, \mathbf{w}_{k}^{+}\right\}_{k=1}^{M}$ of the matrix $\mathcal{A}_{+}$.

2. Normalize the vectors $\mathbf{w}_{k}^{+}$for $k=1, \ldots, M$.

3. Compute the eigenvectors of the matrix $\mathcal{A}_{-}=\mathbf{A}_{+} \mathbf{A}_{-}$,

$$
\mathbf{w}_{k}^{-}=\frac{1}{\lambda_{k}} \mathbf{A}_{+} \mathbf{w}_{k}^{+}, \quad k=1, \ldots, M,
$$

where $\lambda_{k}=\sqrt{\mu_{k}}$ are the positive eigenvalues of the matrix $\mathbf{A}_{m j}$.

4. Set $\mathbf{v}_{k}^{+}=\left(\mathbf{w}_{k}^{+}+\mathbf{w}_{k}^{-}\right) / 2$ and $\mathbf{v}_{k}^{-}=\left(\mathbf{w}_{k}^{+}-\mathbf{w}_{k}^{-}\right) / 2$ for $k=1, \ldots, M$.

5. Construct the eigenvectors of $\mathbf{A}_{m j}$ as

$$
\overline{\mathbf{v}}_{k}^{+}=\left[\begin{array}{c}
\mathbf{v}_{k}^{+} \\
\mathbf{v}_{k}^{-}
\end{array}\right], \quad \overline{\mathbf{v}}_{k}^{-}=\left[\begin{array}{c}
\mathbf{v}_{k}^{-} \\
\mathbf{v}_{k}^{+}
\end{array}\right], \quad k=1, \ldots, M .
$$

The spectral decomposition of the matrix $\mathbf{A}_{m j}$ is then

$$
\mathbf{A}_{m j}=\mathbf{V}_{m j}\left[\begin{array}{cc}
\boldsymbol{\Lambda}_{m j} & \mathbf{0} \\
\mathbf{0} & -\boldsymbol{\Lambda}_{m j}
\end{array}\right] \mathbf{V}_{m j}^{-1}
$$

with

$$
\mathbf{V}_{m, j}=\left[\overline{\mathbf{v}}_{1}^{+}, \ldots, \overline{\mathbf{v}}_{M}^{+}, \overline{\mathbf{v}}_{1}^{-}, \ldots, \overline{\mathbf{v}}_{M}^{-}\right]
$$

and

$$
\Lambda_{m j}=\operatorname{diag}\left[\lambda_{1}, \ldots, \lambda_{M}\right] .
$$

To compute $\partial \mathbf{V}_{m j}^{-1} / \partial \varsigma_{i}$ and $\partial \lambda_{k} / \partial \varsigma_{i}$, we follow the exposition given in [11]. Considering the eigenvalue problem for the matrix $\mathcal{A}_{+}$, i.e.

$$
\mathcal{A}_{+} \mathbf{w}_{k}^{+}=\mu_{k} \mathbf{w}_{k}^{+},
$$

and taking the derivative with respect to $\varsigma_{i}$, we obtain

$$
\frac{\partial \mathcal{A}_{+}}{\partial \varsigma_{i}} \mathbf{w}_{k}^{+}+\mathcal{A}_{+} \frac{\partial \mathbf{w}_{k}^{+}}{\partial \varsigma_{i}}=\frac{\partial \mu_{k}}{\partial \varsigma_{i}} \mathbf{w}_{k}^{+}+\mu_{k} \frac{\partial \mathbf{w}_{k}^{+}}{\partial \varsigma_{i}} .
$$

Equation (63) is a system of $M$ equations with $M+1$ unknowns: the scalar $\partial \mu_{k} / \partial \varsigma_{i}$ and the vector $\partial \mathbf{w}_{k}^{+} / \partial \varsigma_{i}$. Since the eigenvectors $\mathbf{w}_{k}^{+}$are normalized, we derive an additional equation

$$
\mathbf{w}_{k}^{+\top} \frac{\partial \mathbf{w}_{k}^{+}}{\partial \varsigma_{i}}=0,
$$

which yields the compatibility of the system of equations. By (63) and (64), the resulting system of equations can be written in matrix form as

$$
\left[\begin{array}{cc}
\mathbf{w}_{k}^{+} & \mu_{k} \mathbf{I}-\mathcal{A}_{+} \\
0 & \mathbf{w}_{k}^{+\top}
\end{array}\right]\left[\begin{array}{c}
\frac{\partial \mu_{k}}{\partial \varsigma_{i}} \\
\frac{\partial \mathbf{w}_{k}^{+}}{\partial \varsigma_{i}}
\end{array}\right]=\left[\begin{array}{c}
\frac{\partial \mathcal{A}_{+}}{\partial \varsigma_{i}} \mathbf{w}_{k}^{+} \\
0
\end{array}\right] .
$$

It is important to observe that the we can solve the above system of equations for all atmospheric parameters $\varsigma_{i}, i=1, \ldots, N_{\mathrm{p}}$, that is, we can solve the matrix equation

$$
\left[\begin{array}{cc}
\mathbf{w}_{k}^{+} & \mu_{k} \mathbf{I}-\mathcal{A}_{+} \\
0 & \mathbf{w}_{k}^{+\mathbf{T}}
\end{array}\right]\left[\begin{array}{ccc}
\frac{\partial \mu_{k}}{\partial S_{1}} & \cdots & \frac{\partial \mu_{k}}{\partial S_{N_{p}}} \\
\frac{\partial \mathbf{w}_{k}^{+}}{\partial \zeta_{1}} & \cdots & \frac{\partial \mathbf{w}_{k}^{+}}{\partial S_{N_{\mathrm{p}}}}
\end{array}\right]=\left[\begin{array}{ccc}
\frac{\partial \mathcal{A}_{+}}{\partial S_{1}} \mathbf{w}_{k}^{+} & \cdots & \frac{\partial \mathcal{A}_{+}}{\partial S_{N_{\mathrm{p}}}} \mathbf{w}_{k}^{+} \\
0 & \cdots & 0
\end{array}\right]
$$

If $\partial \mu_{k} / \partial \varsigma_{i}$ is known, the partial derivative of $\lambda_{k}=\sqrt{\mu_{k}}$ with respect to $\varsigma_{i}$ follows immediately as

$$
\frac{\partial \lambda_{k}}{\partial \varsigma_{i}}=\frac{1}{2 \lambda_{k}} \frac{\partial \mu_{k}}{\partial \varsigma_{i}}
$$

To compute the partial derivative of $\mathbf{w}_{k}^{-}$, we use definition (57) and apply the chain rule to obtain

$$
\frac{\partial \mathbf{w}_{k}^{-}}{\partial \varsigma_{i}}=\left(-\frac{1}{\lambda_{k}^{2}} \frac{\partial \lambda_{k}}{\partial \varsigma_{i}}\right) \mathbf{A}_{+} \mathbf{w}_{k}^{+}+\frac{1}{\lambda_{k}} \frac{\partial \mathbf{A}_{+}}{\partial \varsigma_{i}} \mathbf{w}_{k}^{+}+\frac{1}{\lambda_{k}} \mathbf{A}_{+} \frac{\partial \mathbf{w}_{k}^{+}}{\partial \varsigma_{i}} .
$$

Further calculations give

$$
\frac{\partial \mathbf{v}_{k}^{+}}{\partial \varsigma_{i}}=\frac{1}{2}\left(\frac{\partial \mathbf{w}_{k}^{+}}{\partial \varsigma_{i}}+\frac{\partial \mathbf{w}_{k}^{-}}{\partial \varsigma_{i}}\right), \quad \frac{\partial \mathbf{v}_{k}^{-}}{\partial \varsigma_{i}}=\frac{1}{2}\left(\frac{\partial \mathbf{w}_{k}^{+}}{\partial \varsigma_{i}}-\frac{\partial \mathbf{w}_{k}^{-}}{\partial \varsigma_{i}}\right),
$$

and

$$
\frac{\partial \overline{\mathbf{v}}_{k}^{+}}{\partial \varsigma_{i}}=\left[\begin{array}{c}
\frac{\partial \mathbf{v}_{k}^{+}}{\partial \varsigma_{i}} \\
\frac{\partial \mathbf{v}_{k}^{-}}{\partial \varsigma_{i}}
\end{array}\right], \quad \frac{\partial \overline{\mathbf{v}}_{k}^{-}}{\partial \varsigma_{i}}=\left[\begin{array}{c}
\frac{\partial \mathbf{v}_{k}^{-}}{\partial \varsigma_{i}} \\
\frac{\partial \mathbf{v}_{k}^{+}}{\partial \varsigma_{i}}
\end{array}\right] .
$$

Using now the definition of $\mathbf{V}_{m j}$ we obtain

$$
\frac{\partial \mathbf{V}_{m j}}{\partial \varsigma_{i}}=\left[\frac{\partial \overline{\mathbf{v}}_{1}^{+}}{\partial \varsigma_{i}}, \ldots, \frac{\partial \overline{\mathbf{v}}_{M}^{+}}{\partial \varsigma_{i}}, \frac{\partial \overline{\mathbf{v}}_{1}^{-}}{\partial \varsigma_{i}}, \ldots, \frac{\partial \overline{\mathbf{v}}_{M}^{-}}{\partial \varsigma_{i}}\right]
$$

whence, taking into account that $\mathbf{V}_{m j} \mathbf{V}_{m j}^{-1}=\mathbf{I}$, we end up with

$$
\frac{\partial \mathbf{V}_{m j}^{-1}}{\partial \varsigma_{i}}=-\mathbf{V}_{m j}^{-1} \frac{\partial \mathbf{V}_{m j}}{\partial \varsigma_{i}} \mathbf{V}_{m j}^{-1}
$$

\section{Appendix B}

In this appendix, we perform the integration over the azimuthal angle in (37) and (38), and discuss the integration over the radial coordinate.

We begin by considering the cosine-azimuthal expansions

$$
I_{\mathrm{d}}(r, \boldsymbol{\Omega})=\sum_{m=0}^{2 M-1} I_{m}(r, \mu) \cos \left[m\left(\varphi-\varphi_{0}\right)\right]
$$

and

$$
\widehat{I_{\mathrm{d}}^{\dagger}}(r, \boldsymbol{\Omega})=\sum_{m=0}^{2 M-1} \widehat{I_{m}^{\dagger}}(r, \mu) \cos \left[m\left(\varphi-\widehat{\varphi}_{\mathrm{m}}\right)\right],
$$

yielding

$$
\widehat{I_{\mathrm{d}}^{\dagger}}(r,-\mathbf{\Omega})=\sum_{m=0}^{2 M-1} \widehat{I_{m}^{\dagger}}(r,-\mu) \cos \left[m\left(\varphi-\varphi_{\mathrm{m}}\right)\right] .
$$


Further, we write

$\sigma_{\mathrm{sct}}(r) P\left(r, \boldsymbol{\Omega}, \boldsymbol{\Omega}^{\prime}\right)=\sum_{m=0}^{2 M-1}\left(2-\delta_{m 0}\right) s_{m}\left(r, \mu, \mu^{\prime}\right) \cos \left[m\left(\varphi-\varphi^{\prime}\right)\right]$,

where (cf. (16) and (17))

$s_{m}\left(r, \mu, \mu^{\prime}\right)=\sigma_{\mathrm{sct}}(r) p_{m}\left(r, \mu, \mu^{\prime}\right)=\sum_{n=m}^{2 M-1} \xi_{n}(r) P_{n}^{m}(\mu) P_{n}^{m}\left(\mu^{\prime}\right)$.

Inserting (73), (75), and (76) in (36)-(38), and using the orthogonality relations of the Fourier cosine basis functions, we obtain

$$
\frac{\partial I_{\mathrm{m}}}{\partial \varsigma_{i}}=\sum_{m=0}^{2 M-1}\left(T_{1 m}+T_{2 m}\right) \cos \left[m\left(\varphi_{\mathrm{m}}-\varphi_{0}\right)\right]
$$

where

$$
\begin{aligned}
& T_{1 m}=-\left(1+\delta_{m 0}\right) \pi\left[\int_{r_{\mathrm{s}}}^{r_{\mathrm{TOA}}} \int_{-1}^{1} \frac{\partial \sigma_{\text {ext }}}{\partial \varsigma_{i}}(r) \widehat{I_{m}^{\dagger}}(r,-\mu) I_{m}(r, \mu) \mathrm{d} r \mathrm{~d} \mu\right] \\
& -F_{0}\left[\int_{r_{\mathrm{s}}}^{r_{\mathrm{TOA}}} \frac{\partial \sigma_{\text {ext }}}{\partial \varsigma_{i}}(r) \widehat{I}_{m}^{\dagger}\left(r, \mu_{0}\right) T(r) \mathrm{d} r\right] \\
& +\frac{1}{2}\left(1+\delta_{m 0}\right) \pi\left[\int_{r_{\mathrm{s}}}^{r_{\text {TOA }}} \int_{-1}^{1} \widehat{I_{m}^{\dagger}}(r,-\mu) \mathrm{d} r \mathrm{~d} \mu \int_{-1}^{1} \frac{\partial s_{m}}{\partial \varsigma_{i}}\left(r, \mu, \mu^{\prime}\right) I_{m}\left(r, \mu^{\prime}\right) \mathrm{d} \mu^{\prime}\right] \\
& +\frac{F_{0}}{2}\left[\int_{r_{\mathrm{s}}}^{r_{\text {TOA }}} \int_{-1}^{1} \frac{\partial s_{m}}{\partial \varsigma_{i}}\left(r, \mu,-\mu_{0}\right) \widehat{I_{m}^{\dagger}}(r,-\mu) T(r) \mathrm{d} r \mathrm{~d} \mu\right]
\end{aligned}
$$

and

$$
\begin{aligned}
T_{2 m} & =-\widehat{F}_{0}\left[\int_{r_{\mathrm{s}}}^{r_{\text {TOA }}} \frac{\partial \sigma_{\text {ext }}}{\partial \varsigma_{i}}(r) I_{m}\left(r, \mu_{\mathrm{m}}\right) \widehat{T}^{\dagger}(r) \mathrm{d} r\right] \\
+ & \frac{\widehat{F}_{0}}{2}\left[\int_{r_{\mathrm{s}}}^{r_{\mathrm{TOA}}} \widehat{T}^{\dagger}(r) \mathrm{d} r \int_{-1}^{1} \frac{\partial s_{m}}{\partial \varsigma_{i}}\left(r, \mu_{\mathrm{m}}, \mu^{\prime}\right) I_{m}\left(r, \mu^{\prime}\right) \mathrm{d} \mu^{\prime}\right] \\
+ & \frac{\widehat{F}_{0} F_{0}}{4 \pi}\left(2-\delta_{m 0}\right)\left[\int_{r_{\mathrm{s}}}^{r_{\mathrm{TOA}}} \frac{\partial s_{m}}{\partial \varsigma_{i}}\left(r, \mu_{\mathrm{m}},-\mu_{0}\right) T(r) \widehat{T}^{\dagger}(r) \mathrm{d} r\right] .
\end{aligned}
$$

To perform the integration over the radial coordinate in (79) and (80), we assume for simplicity that the partial derivatives $\partial \sigma_{\text {ext } j} / \partial \varsigma_{i}$ and $\partial \xi_{n, j} / \partial \varsigma_{i}$ are nonzero for all layers $j \in D_{i}$, where $D_{i}$ is a subset of $\{1, \ldots, N-1\}$. In this case, the integration with respect to the radial coordinate reduces to an integration over all layers in $D_{i}$; we have

$$
\int_{r_{\mathrm{s}}}^{r_{\mathrm{TOA}}} \mathrm{d} r=\sum_{j \in D_{i}} \int_{r_{j+1}}^{r_{j}} \mathrm{~d} r
$$

For the azimuthal mode $m$, let $I_{m j}\left(\rho, \pm \mu_{k}\right)$ and $\widehat{I}_{m j}^{\dagger}\left(\rho, \pm \mu_{k}\right)$ be, respectively, the forward and conjugate adjoint radiances at an internal point $\rho$ in the layer $j\left(0 \leq \rho \leq \Delta r_{j}\right)$. Using the analytic representation of the radiance at an internal layer point as given in [23], the integrals

$$
\begin{aligned}
\mathcal{I}_{m j}(\mu) & =\int_{0}^{\Delta r_{j}} I_{m j}(\rho, \mu) \widehat{T}^{\dagger}(\rho) \mathrm{d} \rho \\
& =\int_{0}^{\Delta r_{j}} \mathrm{e}^{-\left[\frac{\rho}{\Delta r_{j}} \tau_{\text {ext }}^{\mathrm{m}}+\left(1-\frac{\rho}{\Delta r_{j}}\right) \tau_{\text {ext } \mathrm{m}+1}^{\mathrm{m}}\right]} I_{m j}(\rho, \mu) \mathrm{d} \rho, \\
\widehat{\mathcal{I}}_{m j}(\mu) & =\int_{0}^{\Delta r_{j}} \widehat{I}_{m j}^{\dagger}(\rho, \mu) T(\rho) \mathrm{d} \rho \\
& =\int_{0}^{\Delta r_{j}} \mathrm{e}^{-\left[\frac{\rho}{\Delta r_{j}} \tau_{\text {ext }}^{0}+\left(1-\frac{\rho}{\Delta r_{j}}\right) \tau_{\text {ext } j+1}^{0}\right]} \widehat{I}_{m j}^{\dagger}(\rho, \mu) \mathrm{d} \rho, \\
\mathcal{J}_{m j}\left(\mu, \mu^{\prime}\right) & =\int_{0}^{\Delta r_{j}} \widehat{I}_{m j}^{\dagger}(\rho, \mu) I_{m j}\left(\rho, \mu^{\prime}\right) \mathrm{d} \rho,
\end{aligned}
$$

for $\mu= \pm \mu_{k}, \mu^{\prime}= \pm \mu_{l}, k, l=1, \ldots, M$, as well as the integral

$$
\begin{aligned}
\mathcal{T}_{j} & =\int_{0}^{\Delta r_{j}} T(\rho) \widehat{T}^{\dagger}(\rho) \mathrm{d} \rho \\
& =\int_{0}^{\Delta r_{j}} \mathrm{e}^{-\left[\frac{\rho}{\Delta r_{j}}\left(\tau_{\text {ext } j}^{0}+\tau_{\text {ext } j}^{\mathrm{m}}\right)+\left(1-\frac{\rho}{\Delta r_{j}}\right)\left(\tau_{\text {ext } j+1}^{0}+\tau_{\text {ext } j+1}^{\mathrm{m}}\right)\right]} \mathrm{d} \rho,
\end{aligned}
$$

can be computed analytically. Here, $\tau_{\text {ext } j}^{0}$ and $\tau_{\text {ext } j+1}^{0}$ are the optical depths along the geometrical characteristic $\boldsymbol{\Omega}_{0}=$ $\left(-\mu_{0}, \varphi_{0}\right)$ at the boundary levels $j$ and $j+1$, respectively, while $\tau_{\text {ext } j}^{\mathrm{m}}$ and $\tau_{\text {ext } j+1}^{\mathrm{m}}$ are the optical depths along the geometrical characteristic $\widehat{\boldsymbol{\Omega}}_{\mathrm{m}}=\left(\widehat{\mu}_{\mathrm{m}}, \widehat{\varphi}_{\mathrm{m}}\right)=\left(-\mu_{\mathrm{m}}, \varphi_{\mathrm{m}}+\pi\right)$ at the boundary levels $j$ and $j+1$, respectively. We end up with

$$
\begin{aligned}
T_{1 m} & =-\left(1+\delta_{m 0}\right) \pi \sum_{j \in D_{i}} T_{1 m j}^{a}-F_{0} \sum_{j \in D_{i}} T_{1 m j}^{b} \\
& -\frac{1}{2}\left(1+\delta_{m 0}\right) \pi \sum_{j \in D_{i}} T_{1 m j}^{c}+\frac{F_{0}}{2} \sum_{j \in D_{i}} T_{1 m j}^{d}, \\
T_{2 m} & =-\widehat{F}_{0} \sum_{j \in D_{i}} T_{2 m j}^{a}+\frac{\widehat{F}_{0}}{2} \sum_{j \in D_{i}} T_{2 m j}^{b} \\
& +\frac{\widehat{F}_{0} F_{0}}{4 \pi}\left(2-\delta_{m 0}\right) \sum_{j \in D_{i}} T_{2 m j}^{c},
\end{aligned}
$$

where

$$
\begin{aligned}
T_{1 m j}^{a} & =\frac{\partial \sigma_{\mathrm{ext} j}}{\partial \varsigma_{i}} \sum_{k} w_{k}\left[\mathcal{J}_{m j}\left(-\mu_{k}, \mu_{k}\right)+\mathcal{J}_{m j}\left(\mu_{k},-\mu_{k}\right)\right] \\
T_{1 m j}^{b} & =\frac{\partial \sigma_{\mathrm{ext} j}}{\partial \varsigma_{i}} \widehat{\mathcal{I}}_{m j}\left(\mu_{0}\right) \\
T_{1 m j}^{c} & =\sum_{l} \sum_{k} w_{l} w_{k}\left[\frac{\partial s_{m j}}{\partial \varsigma_{i}}\left(\mu_{l}, \mu_{k}\right) \mathcal{J}_{m j}\left(-\mu_{l}, \mu_{k}\right)\right. \\
& +\frac{\partial s_{m j}}{\partial \varsigma_{i}}\left(\mu_{l},-\mu_{k}\right) \mathcal{J}_{m j}\left(-\mu_{l},-\mu_{k}\right)+\frac{\partial s_{m j}}{\partial \varsigma_{i}}\left(-\mu_{l}, \mu_{k}\right) \mathcal{J}_{m j}\left(\mu_{l}, \mu_{k}\right) \\
& \left.+\frac{\partial s_{m j}}{\partial \varsigma_{i}}\left(-\mu_{l},-\mu_{k}\right) \mathcal{J}_{m j}\left(\mu_{l},-\mu_{k}\right)\right] \\
T_{1 m j}^{d} & =\sum_{k} w_{k}\left[\frac{\partial s_{m j}}{\partial \varsigma_{i}}\left(\mu_{k},-\mu_{0}\right) \widehat{\mathcal{I}}_{m j}\left(-\mu_{k}\right)\right. \\
& \left.+\frac{\partial s_{m j}}{\partial \varsigma_{i}}\left(-\mu_{k},-\mu_{0}\right) \widehat{\mathcal{I}}_{m j}\left(\mu_{k}\right)\right]
\end{aligned}
$$


and

$$
\begin{aligned}
T_{2 m j}^{a} & =\frac{\partial \sigma_{\mathrm{ext} j}}{\partial \varsigma_{i}} \mathcal{I}_{m j}\left(\mu_{\mathrm{m}}\right) \\
T_{2 m j}^{b} & =\sum_{k} w_{k}\left[\frac{\partial s_{m j}}{\partial \varsigma_{i}}\left(\mu_{\mathrm{m}}, \mu_{k}\right) \mathcal{I}_{m j}\left(\mu_{k}\right)\right. \\
& \left.+\frac{\partial s_{m j}}{\partial \varsigma_{i}}\left(\mu_{\mathrm{m}},-\mu_{k}\right) \mathcal{I}_{m j}\left(-\mu_{k}\right)\right] \\
T_{2 m j}^{c} & =\frac{\partial s_{m j}}{\partial \varsigma_{i}}\left(\mu_{\mathrm{m}},-\mu_{0}\right) \mathcal{T}_{j} .
\end{aligned}
$$

The integrals $\widehat{\mathcal{I}}_{m j}\left(\mu_{0}\right)$ and $\mathcal{I}_{m j}\left(\mu_{\mathrm{m}}\right)$, which enter the expressions of $T_{1 m j}^{b}$ and $T_{2 m j}^{a}$, respectively, can be computed by using the method of false discrete ordinate (i.e. the false discrete ordinates $\mu_{0}$ and $\mu_{\mathrm{m}}$ with zero weights are added to the set $\left\{\mu_{k}\right\}_{k=1}^{M}$ ).

\section{Appendix C}

In this appendix, we present the first discretization method of Section 3.3, which consists of the discretization of the atmosphere above, below and in the cloud. The linearization method pertinent to this discretization scheme is the forwardadjoint approach. Let us consider an homogeneous cloud with top height $h_{\mathrm{t}}$ and bottom height $h_{\mathrm{b}}$, and let the optical properties of the homogeneous cloud be described by the cloud extinction coefficient $\sigma_{\text {ext }}^{\mathrm{c}}$ and the expansion coefficients $\xi_{n}^{\mathrm{c}}$, so that $s_{m}^{\mathrm{c}}\left(\mu, \mu^{\prime}\right)=\sum_{n=m}^{2 M-1} \xi_{n}^{\mathrm{c}} P_{n}^{m}(\mu) P_{n}^{m}\left(\mu^{\prime}\right)$. Set

$$
\begin{aligned}
\sigma_{\mathrm{ext}}(r) & =\sigma_{\mathrm{ext}}^{0}(r)+H\left(h_{\mathrm{t}}-r\right) H\left(r-h_{\mathrm{b}}\right) \sigma_{\mathrm{ext}}^{\mathrm{c}}, \\
s_{m}\left(r, \mu, \mu^{\prime}\right) & =s_{m}^{0}\left(r, \mu, \mu^{\prime}\right)+H\left(h_{\mathrm{t}}-r\right) H\left(r-h_{\mathrm{b}}\right) s_{m}^{\mathrm{c}}\left(\mu, \mu^{\prime}\right),
\end{aligned}
$$

where the superscript " 0 " refers to the clear sky atmosphere, so that from

$$
\frac{\partial H\left(h_{\mathrm{t}}-r\right)}{\partial h_{\mathrm{t}}}=\delta\left(h_{\mathrm{t}}-r\right)
$$

we get

$$
\begin{gathered}
\frac{\partial \sigma_{\text {ext }}}{\partial h_{\mathrm{t}}}(r)=\delta\left(h_{\mathrm{t}}-r\right) H\left(r-h_{\mathrm{b}}\right) \sigma_{\text {ext }}^{\mathrm{c}}, \\
\frac{\partial s_{m}}{\partial h_{\mathrm{t}}}\left(r, \mu, \mu^{\prime}\right)=\delta\left(h_{\mathrm{t}}-r\right) H\left(r-h_{\mathrm{b}}\right) s_{m}^{\mathrm{c}}\left(\mu, \mu^{\prime}\right) .
\end{gathered}
$$

Inserting (97) and (98) in (79) and (80), we find, for example, that the first integral in (79) is given by

$$
\begin{gathered}
\int_{r_{\mathrm{s}}}^{r_{\mathrm{TOA}}} \int_{-1}^{1} \frac{\partial \sigma_{\text {ext }}(r)}{\partial \varsigma_{i}} \widehat{I}_{m}^{\dagger}(r,-\mu) I_{m}(r, \mu) \mathrm{d} r \mathrm{~d} \mu \\
\quad=\int_{-1}^{1} \sigma_{\text {ext }}^{\mathrm{c}} \widehat{I_{m}^{\dagger}}\left(h_{\mathrm{t}},-\mu\right) I_{m}\left(h_{\mathrm{t}}, \mu\right) \mathrm{d} \mu
\end{gathered}
$$

and that the third integral in (79) is given by

$$
\begin{gathered}
\int_{r_{\mathrm{s}}}^{r_{\mathrm{TOA}}} \int_{-1}^{1} \widehat{I_{m}^{\dagger}}(r,-\mu) \mathrm{d} r \mathrm{~d} \mu \int_{-1}^{1} \frac{\partial s_{m}}{\partial \varsigma_{i}}\left(r, \mu, \mu^{\prime}\right) I_{m}\left(r, \mu^{\prime}\right) \mathrm{d} \mu^{\prime} \\
=\int_{-1}^{1} \widehat{I_{m}^{\dagger}}\left(h_{\mathrm{t}},-\mu\right) \mathrm{d} \mu \int_{-1}^{1} s_{m}^{\mathrm{c}}\left(\mu, \mu^{\prime}\right) I_{m}\left(h_{\mathrm{t}}, \mu^{\prime}\right) \mathrm{d} \mu^{\prime} .
\end{gathered}
$$

For the derivatives with respect to the cloud bottom height $h_{\mathrm{b}}$, we use

$$
\frac{\partial H\left(r-h_{\mathrm{b}}\right)}{\partial h_{\mathrm{b}}}=-\delta\left(r-h_{\mathrm{b}}\right)
$$

and proceed analogously.

\section{References}

[1] A. Marshak, Y. Knyazikhin, The spectral invariant approximation within canopy radiative transfer to support the use of the EPIC/DSCOVR oxygen B-band for monitoring vegetation, Journal of Quantitative Spectroscopy and Radiative Transfer 191 (2017) 7-12. doi:10.1016/j.jqsrt.2017.01.015

[2] X. Xu, J. Wang, Y. Wang, J. Zeng, O. Torres, Y. Yang, A. Marshak, J. Reid, S. Miller, Passive remote sensing of altitude and optical depth of dust plumes using the oxygen A and B bands: First results from EPIC/DSCOVR at Lagrange-1 point, Geophysical Research Letters 44 (14) (2017) 7544-7554. doi: $10.1002 / 2017 \mathrm{~g} 1073939$

[3] Y. Yang, A. Marshak, J. Mao, A. Lyapustin, J. Herman, A method of retrieving cloud top height and cloud geometrical thickness with oxygen A and B bands for the Deep Space Climate Observatory (DSCOVR) mission: Radiative transfer simulations, Journal of Quantitative Spectroscopy and Radiative Transfer 122 (2013) 141-149. doi:10.1016/j.jqsrt.2012.09.017

[4] V. Molina García, S. Sasi, D. Efremenko, A. Doicu, D. Loyola, Radiative transfer models for retrieval of cloud parameters from EPIC/DSCOVR measurements, Journal of Quantitative Spectroscopy and Radiative Transfer 123 (2018) 228-240. doi:10.1016/j.jqsrt.2018.03.014

[5] R. Goody, R. West, L. Chen, D. Crisp, The correlated k-method for radiation calculations in nonhomogeneous atmospheres, Journal of Quantitative Spectroscopy and Radiative Transfer 42 (6) (1989) 539-550. doi: 10.1016/0022-4073(89) 90044-7

[6] V. Natraj, X. Jiang, R. Shia, X. Huang, J. Margolis, Y. Yung, Application of the principal component analysis to high spectral resolution radiative transfer: A case study of the $\mathrm{O}_{2}$ A-band, Journal of Quantitative Spectroscopy and Radiative Transfer 95 (4) (2005) 539-556. doi:10.1016/j.jqsrt.2004.12.024

[7] V. Natraj, R. Shia, Y. Yung, On the use of principal component analysis to speed up radiative transfer calculations, Journal of Quantitative Spectroscopy and Radiative Transfer 111 (5) (2010) 810-816. doi:10.1016/j.jqsrt.2009.11.004

[8] R. Spurr, V. Natraj, C. Lerot, V. Roozendael, D. Loyola, Linearization of the principal component analysis method for radiative transfer acceleration: Application to retrieval algorithms and sensitivity studies, Journal of Quantitative Spectroscopy and Radiative Transfer 125 (2013) 1-17. doi:10.1016/j.jqsrt.2013.04.002

[9] D. Efremenko, A. Doicu, D. Loyola, T. Trautmann, Optical property dimensionality reduction techniques for accelerated radiative transfer performance: Application to remote sensing total ozone retrievals, Journal of Quantitative Spectroscopy and Radiative Transfer 133 (2014) 128-135. doi:10.1016/j.jqsrt.2013.07.023

[10] R. Spurr, T. Kurosu, K. Chance, A linearized discrete ordinate radiative transfer model for atmospheric remote-sensing retrieval, Journal of Quantitative Spectroscopy and Radiative Transfer 68 (6) (2001) 689-735. doi: 10.1016/s0022-4073(00)00055-8

[11] R. Spurr, Simultaneous derivation of intensities and weighting functions in a general pseudo-spherical discrete ordinate radiative transfer treatment, Journal of Quantitative Spectroscopy and Radiative Transfer 75 (2) (2002) 129-175. doi: 10.1016/s0022-4073(01)00245-x

[12] R. J. D. Spurr, LIDORT and VLIDORT. Linearized pseudo-spherical scalar and vector discrete ordinate radiative transfer models for use in remote sensing retrieval problems, in: A. Kokhanovsky (Ed.), Light scattering reviews, Vol. 3, 2008, pp. 229-275. doi: $10.1007 / 978-3-540-48546-9 \_7$ 
[13] R. Spurr, M. Christi, Linearization of the interaction principle: Analytic jacobians in the "radiant model", Journal of Quantitative Spectroscopy and Radiative Transfer 103 (3) (2007) 431-446.

doi:10.1016/j.jqsrt.2006.05.001

[14] G. Marchuk, Equation for the value of information from weather satellites and formulation of inverse problems, Cosmic Research (3) (1964) 462477, [in Russian].

[15] G. Marchuk, Adjoint equations and analysis of complex systems, Springer Nature, 1995. doi:10.1007/978-94-017-0621-6

[16] M. Box, Radiative perturbation theory: A review, Environmental Modelling \& Software 17 (1) (2002) 95-106. doi:10.1016/s1364-8152(01)00056-1

[17] E. Ustinov, Adjoint sensitivity analysis of radiative transfer equation: temperature and gas mixing ratio weighting functions for remote sensing of scattering atmospheres in thermal IR, Journal of Quantitative Spectroscopy and Radiative Transfer 68 (2) (2001) 195-211. doi:10.1016/s0022-4073(00)00022-4

[18] E. Ustinov, Atmospheric weighting functions and surface partial derivatives for remote sensing of scattering planetary atmospheres in thermal spectral region: General adjoint approach, Journal of Quantitative Spectroscopy and Radiative Transfer 92 (3) (2005) 351-371. doi:10.1016/j.jqsrt.2004.08.003

[19] V. Rozanov, A. Rozanov, Relationship between different approaches to derive weighting functions related to atmospheric remote sensing problems, Journal of Quantitative Spectroscopy and Radiative Transfer 105 (2) (2007) 217-242. doi:10.1016/j.jqsrt.2006.12.006

[20] A. Doicu, T. Trautmann, Adjoint problem of radiative transfer for a pseudo-spherical atmosphere and general viewing geometries, Journal of Quantitative Spectroscopy and Radiative Transfer 110 (8) (2009) 464476.

doi:10.1016/j.jqsrt.2009.01.027

[21] J. Landgraf, O. Hasekamp, M. Box, T. Trautmann, A linearized radiative transfer model for ozone profile retrieval using the analytical forwardadjoint perturbation theory approach, Journal of Geophysical Research: Atmospheres 106 (D21) (2001) 27291-27305. doi: $10.1029 / 2001$ jd000636

[22] A. Doicu, T. Trautmann, Two linearization methods for atmospheric remote sensing, Journal of Quantitative Spectroscopy and Radiative Transfer 110 (8) (2009) 477-490. doi:10.1016/j.jqsrt.2009.02.001

[23] A. Doicu, T. Trautmann, Discrete-ordinate method with matrix exponential for a pseudo-spherical atmosphere: Scalar case, Journal of Quantitative Spectroscopy and Radiative Transfer 110 (1-2) (2009) 146-158. doi:10.1016/j.jqsrt.2008.09.014

[24] A. Doicu, T. Trautmann, Discrete-ordinate method with matrix exponential for a pseudo-spherical atmosphere: Vector case, Journal of Quantitative Spectroscopy and Radiative Transfer 110 (1-2) (2009) 159-172. doi:10.1016/j.jqsrt.2008.09.013

[25] Q. Liu, F. Weng, Advanced doubling-adding method for radiative transfer in planetary atmospheres, Journal of the Atmospheric Sciences 63 (12) (2006) 3459-3465.

doi: $10.1175 /$ jas3808.1

[26] W. Wiscombe, The delta-M method: Rapid yet accurate radiative flux calculations for strongly asymmetric phase functions, Journal of the Atmospheric Sciences 34 (9) (1977) 1408-1422. doi:10.1175/1520-0469(1977)034<1408:tdmrya>2.0.co;2

[27] T. Nakajima, M. Tanaka, Algorithms for radiative intensity calculations in moderately thick atmospheres using a truncation approximation, Journal of Quantitative Spectroscopy and Radiative Transfer 40 (1) (1988) 5169. doi:10.1016/0022-4073(88)90031-3

[28] K. Evans, G. Stephens, A new polarized atmospheric radiative transfer model, Journal of Quantitative Spectroscopy and Radiative Transfer 46 (5) (1991) 413-423. doi:10.1016/0022-4073(91)90043-p

[29] E. Chalhoub, R. Garcia, The equivalence between two techniques of angular interpolation for the discrete-ordinates method, Journal of Quantitative Spectroscopy and Radiative Transfer 64 (5) (2000) 517-535. doi : 10.1016/s0022-4073(99)00134-x
[30] D. Efremenko, A. Doicu, D. Loyola, T. Trautmann, Acceleration techniques for the discrete ordinate method, Journal of Quantitative Spectroscopy and Radiative Transfer 114 (2013) 73-81.

doi:10.1016/j.jqsrt.2012.08.014

[31] F. Schreier, S. Gimeno García, P. Hedelt, M. Hess, J. Mendrok, M. Vasquez, J. Xu, GARLIC - A general purpose atmospheric radiative transfer line-by-line infrared-microwave code: Implementation and evaluation, Journal of Quantitative Spectroscopy and Radiative Transfer 137 (2014) 29-50. doi:10.1016/j.jqsrt.2013.11.018

[32] F. Schreier, Optimized implementations of rational approximations for the Voigt and complex error function, Journal of Quantitative Spectroscopy and Radiative Transfer 112 (6) (2011) 1010-1025. doi: $10.1016 / j \cdot j q s r t .2010 .12 .010$

[33] R. Spurr, J. Wang, J. Zeng, M. Mishchenko, Linearized T-matrix and Mie scattering computations, Journal of Quantitative Spectroscopy and Radiative Transfer 113 (6) (2012) 425-439. doi:10.1016/j.jqsrt.2011.11.014 